ESAIM: M2AN

Vol. 40, $\mathrm{N}^{\circ} 1,2006$, pp. 1-28

DOI: $10.1051 / \mathrm{m} 2 \mathrm{an}: 2006003$
ESAIM: Mathematical Modelling and Numerical Analysis

www.edpsciences.org/m2an

\title{
ANALYSIS OF A NEW AUGMENTED MIXED FINITE ELEMENT METHOD FOR LINEAR ELASTICITY ALLOWING $\mathbb{R T}_{0}-\mathbb{P}_{1}-\mathbb{P}_{0}$ APPROXIMATIONS *
}

\author{
GABRIEL N. GaticA ${ }^{1}$
}

\begin{abstract}
We present a new stabilized mixed finite element method for the linear elasticity problem in $\mathbb{R}^{2}$. The approach is based on the introduction of Galerkin least-squares terms arising from the constitutive and equilibrium equations, and from the relation defining the rotation in terms of the displacement. We show that the resulting augmented variational formulation and the associated Galerkin scheme are well posed, and that the latter becomes locking-free and asymptotically locking-free for Dirichlet and mixed boundary conditions, respectively. In particular, the discrete scheme allows the utilization of Raviart-Thomas spaces of lowest order for the stress tensor, piecewise linear elements for the displacement, and piecewise constants for the rotation. In the case of mixed boundary conditions, the essential one (Neumann) is imposed weakly, which yields the introduction of the trace of the displacement as a suitable Lagrange multiplier. This trace is then approximated by piecewise linear elements on an independent partition of the Neumann boundary whose mesh size needs to satisfy a compatibility condition with the mesh size associated to the triangulation of the domain. Several numerical results illustrating the good performance of the augmented mixed finite element scheme in the case of Dirichlet boundary conditions are also reported.
\end{abstract}

Mathematics Subject Classification. 65N12, 65N15, 65N30, 74 B05.

Received: December 4, 2004.

\section{INTRODUCTION}

The possibility of introducing further unknowns of physical interest, such as stresses and rotations, and the need of locking-free numerical schemes have strongly motivated the utilization of dual-mixed variational formulations and the associated mixed finite element methods in linear and nonlinear elasticity (see, e.g. [6,8,10], and the references therein). The additional unknowns are then approximated directly, which avoids the numerical postprocessing that is usually employed with the solutions arising from primal formulations. Consequently, the derivation of appropriate finite element subspaces yielding well posed Galerkin schemes and a priori error estimates has been extensively studied and several choices are already available in the literature. In particular, for linear plane elasticity we refer to the family presented in [32], which includes the classical PEERS element from [1] and a modification of the $\mathrm{BDM}_{k}$ spaces (see $[10,12,13]$ ). On the other hand, a possibility that has

\footnotetext{
Keywords and phrases. Mixed-FEM, augmented formulation, linear elasticity, locking-free.

* This research was partially supported by CONICYT-Chile through the FONDAP Program in Applied Mathematics, and by the Dirección de Investigación of the Universidad de Concepción through the Advanced Research Groups Program.

$1 \mathrm{GI}^{2} \mathrm{MA}$, Departamento de Ingeniería Matemática, Universidad de Concepción, Casilla 160-C, Concepción, Chile. ggatica@ing-mat.udec.cl
} 
also been widely investigated is the stabilization of dual-mixed variational formulations through the application of diverse procedures. To this respect, we first mention $[30,33,34]$ in which a locally stabilized mixed finite element formulation, a stabilized hybrid scheme, and a locking-free mixed finite element scheme with continuous displacement, respectively, are proposed and analyzed. A quite general technique, certainly not restricted to dual-mixed schemes, is given by the augmented variational formulations, which go back to [19,20]. They are also known as Galerkin least-squares methods and have already been extended in different directions. Some applications to elasticity problems can be found in [14,22], and a non-symmetric variant was considered in [17] for the Stokes problem. In addition, stabilized mixed finite element methods for related problems, including Darcy flow, incompressible flows, plates, and shells, can be seen in $[2,9,15,18,21,26,27,29,31]$. The stabilizing terms employed there consider, among others, interelement jumps, bubble functions, and local residuals. For an abstract framework concerning the stabilization of general mixed finite element methods, we refer to [11].

The purpose of this work is to present a new locking-free stabilized mixed finite element scheme for the linear elasticity problem in the plane. It is based on the introduction of suitable Galerkin least-squares terms arising from the constitutive and equilibrium equations, and from the relation connecting the rotation with the displacement. Our method, which yields an augmented variational formulation and is easily generalized to $3 \mathrm{D}$, can be viewed as an adaptation to the present case of the non-symmetric procedures utilized in [17, 29]. A similar approach for second order elliptic equations in divergence form, which includes a boundary residual term expressed in the $H^{1 / 2}$ Sobolev norm by means of wavelet bases, is proposed in [7]. The rest of the paper is organized as follows. In Section 2 we describe the boundary value problems of interest and provide the corresponding dual-mixed variational formulations with Dirichlet and mixed boundary conditions. In Section 3 we introduce the augmented continuous formulations and show that they are well posed. The lockingfree augmented Galerkin schemes are analyzed in Section 4 and explicit finite element subspaces providing unique solvability, a priori estimates, and convergence, are defined there. Some computational aspects are also discussed. As a general remark, we realize that the usual trick of dealing, "for simplicity of the presentation", with pure Dirichlet conditions, actually aims to hide the further difficulties arising with the presence of nonhomogeneous Neumann data. In particular, we show that in the case of mixed boundary conditions, the locking-free property of the discrete scheme only holds asymptotically, that is for sufficiently small mesh sizes. Finally, in Section 5 we present some numerical results for the case of pure Dirichlet boundary conditions.

We end this section with some notations to be used below. Given any Hilbert space $U, U^{2}$ and $U^{2 \times 2}$ denote, respectively, the space of vectors and square matrices of order 2 with entries in $U$. In particular, $\mathbf{I}$ is the identity matrix of $\mathbb{R}^{2 \times 2}$, and given $\boldsymbol{\tau}:=\left(\tau_{i j}\right), \boldsymbol{\zeta}:=\left(\zeta_{i j}\right) \in \mathbb{R}^{2 \times 2}$, we write as usual $\boldsymbol{\tau}^{\mathrm{t}}:=\left(\tau_{j i}\right), \quad \operatorname{tr}(\boldsymbol{\tau}):=$ $\sum_{i=1}^{2} \tau_{i i}, \quad \boldsymbol{\tau}^{\mathrm{d}}:=\boldsymbol{\tau}-\frac{1}{2} \operatorname{tr}(\boldsymbol{\tau}) \mathbf{I}, \quad$ and $\boldsymbol{\tau}: \boldsymbol{\zeta}:=\sum_{i, j=1}^{2} \tau_{i j} \zeta_{i j}$. Also, in what follows we utilize the standard terminology for Sobolev spaces and norms, and use $C$ and $c$, with or without subscripts, bars, tildes or hats, to denote generic constants independent of the discretization parameters, which may take different values at different places.

\section{The BOUndary VAlue PROBlems}

Let $\Omega$ be a bounded and simply connected domain in $\mathbb{R}^{2}$ with Lipschitz-continuous boundary $\Gamma$, and let $\Gamma_{D}$ and $\Gamma_{N}$ be two disjoint subsets of $\Gamma$ such that $\left|\Gamma_{D}\right| \neq 0$ and $\Gamma=\bar{\Gamma}_{D} \cup \bar{\Gamma}_{N}$. Our goal is to determine the displacement $\mathbf{u}$ and stress tensor $\boldsymbol{\sigma}$ of a linear elastic material occupying the region $\Omega$. In other words, given a volume force $\mathbf{f} \in\left[L^{2}(\Omega)\right]^{2}$ and a traction $\mathbf{g} \in\left[H^{-1 / 2}\left(\Gamma_{N}\right)\right]^{2}$, we seek a symmetric tensor field $\boldsymbol{\sigma}$ and a vector field $\mathbf{u}$ such that

$$
\begin{gathered}
\boldsymbol{\sigma}=\mathcal{C} \mathbf{e}(\mathbf{u}), \quad \operatorname{div}(\boldsymbol{\sigma})=-\mathbf{f} \text { in } \Omega, \\
\mathbf{u}=0 \quad \text { on } \Gamma_{D}, \quad \text { and } \boldsymbol{\sigma} \boldsymbol{\nu}=\mathbf{g} \text { on } \Gamma_{N} .
\end{gathered}
$$

Hereafter, $\mathbf{e}(\mathbf{u}):=\frac{1}{2}\left(\nabla \mathbf{u}+(\nabla \mathbf{u})^{\mathrm{t}}\right)$ is the strain tensor of small deformations, $\boldsymbol{\nu}$ is the unit outward normal to $\Gamma$, and $\mathcal{C}$ is the elasticity tensor determined by Hooke's law, that is

$$
\mathcal{C} \boldsymbol{\zeta}:=\lambda \operatorname{tr}(\boldsymbol{\zeta}) \mathbf{I}+2 \mu \boldsymbol{\zeta} \quad \forall \boldsymbol{\zeta} \in\left[L^{2}(\Omega)\right]^{2 \times 2},
$$


where $\lambda, \mu>0$ denote the corresponding Lamé constants. It is easy to see from (2.2) that the inverse tensor $\mathcal{C}^{-1}$ reduces to

$$
\mathcal{C}^{-1} \boldsymbol{\zeta}:=\frac{1}{2 \mu} \boldsymbol{\zeta}-\frac{\lambda}{4 \mu(\lambda+\mu)} \operatorname{tr}(\boldsymbol{\zeta}) \mathbf{I} \quad \forall \boldsymbol{\zeta} \in\left[L^{2}(\Omega)\right]^{2 \times 2} .
$$

\subsection{Dirichlet boundary conditions}

We are certainly interested in the mixed method of Hellinger and Reissner since it provides simultaneous approximations of $\mathbf{u}$ and $\boldsymbol{\sigma}$. More precisely, imposing weakly the symmetry of $\boldsymbol{\sigma}$ through the introduction of the rotation $\gamma:=\frac{1}{2}\left(\nabla \mathbf{u}-(\nabla \mathbf{u})^{\mathrm{t}}\right)$ as a further unknown (see $[1,32]$ ), multiplying by tests functions and then integrating the equilibrium equation and the relation $\nabla \mathbf{u}-\boldsymbol{\gamma}=\mathbf{e}(\mathbf{u})=\mathcal{C}^{-1} \boldsymbol{\sigma}$ (see (2.3)), we end up with the following dual-mixed variational formulation of $(2.1)-(2.2)$ in the case of pure Dirichlet boundary conditions: find $(\boldsymbol{\sigma},(\mathbf{u}, \boldsymbol{\gamma})) \in H \times Q$ such that

$$
\begin{aligned}
a(\boldsymbol{\sigma}, \boldsymbol{\tau})+b(\boldsymbol{\tau},(\mathbf{u}, \boldsymbol{\gamma})) & =0 \quad \forall \boldsymbol{\tau} \in H, \\
b(\boldsymbol{\sigma},(\mathbf{v}, \boldsymbol{\eta})) & =-\int_{\Omega} \mathbf{f} \cdot \mathbf{v} \quad \forall(\mathbf{v}, \boldsymbol{\eta}) \in Q,
\end{aligned}
$$

where $H=H(\operatorname{div} ; \Omega):=\left\{\boldsymbol{\tau} \in\left[L^{2}(\Omega)\right]^{2 \times 2}: \operatorname{div}(\boldsymbol{\tau}) \in\left[L^{2}(\Omega)\right]^{2}\right\}, Q:=\left[L^{2}(\Omega)\right]^{2} \times\left[L^{2}(\Omega)\right]_{\text {skew }}^{2 \times 2}$, with $\left[L^{2}(\Omega)\right]_{\text {skew }}^{2 \times 2}:=\left\{\boldsymbol{\eta} \in\left[L^{2}(\Omega)\right]^{2 \times 2}: \quad \boldsymbol{\eta}+\boldsymbol{\eta}^{\mathrm{t}}=0\right\}$, and the bilinear forms $a: H \times H \rightarrow \mathbb{R}$ and $b: H \times Q \rightarrow \mathbb{R}$ are defined by

and

$$
a(\boldsymbol{\zeta}, \boldsymbol{\tau}):=\int_{\Omega} \mathcal{C}^{-1} \boldsymbol{\zeta}: \boldsymbol{\tau}=\frac{1}{2 \mu} \int_{\Omega} \boldsymbol{\zeta}: \boldsymbol{\tau}-\frac{\lambda}{4 \mu(\lambda+\mu)} \int_{\Omega} \operatorname{tr}(\boldsymbol{\zeta}) \operatorname{tr}(\boldsymbol{\tau})
$$

$$
b(\boldsymbol{\tau},(\mathbf{v}, \boldsymbol{\eta})):=\int_{\Omega} \mathbf{v} \cdot \operatorname{div}(\boldsymbol{\tau})+\int_{\Omega} \boldsymbol{\eta}: \boldsymbol{\tau}
$$

for all $\boldsymbol{\zeta}, \boldsymbol{\tau} \in H$ and for all $(\mathbf{v}, \boldsymbol{\eta}) \in Q$.

It is easy to see from $(2.5)$ and $(2.6)$ that for any $(\boldsymbol{\tau},(\mathbf{v}, \boldsymbol{\eta}), c) \in\left[L^{2}(\Omega)\right]^{2 \times 2} \times Q \times \mathbb{R}$ there holds

$$
a(c \mathbf{I}, \boldsymbol{\tau})=\frac{c}{2(\lambda+\mu)} \int_{\Omega} \operatorname{tr}(\boldsymbol{\tau}) \text { and } b(c \mathbf{I},(\mathbf{v}, \boldsymbol{\eta}))=0 .
$$

Also, it is important to remark that $a$ can be rewritten as

$$
a(\boldsymbol{\zeta}, \boldsymbol{\tau})=\frac{1}{2 \mu} \int_{\Omega} \boldsymbol{\zeta}^{\mathrm{d}}: \boldsymbol{\tau}^{\mathrm{d}}+\frac{1}{4(\lambda+\mu)} \int_{\Omega} \operatorname{tr}(\boldsymbol{\zeta}) \operatorname{tr}(\boldsymbol{\tau}),
$$

which implies that

$$
a(\boldsymbol{\tau}, \boldsymbol{\tau}) \geq \frac{1}{2 \mu}\left\|\boldsymbol{\tau}^{\mathrm{d}}\right\|_{\left[L^{2}(\Omega)\right]^{2 \times 2}}^{2} \quad \forall \boldsymbol{\tau} \in\left[L^{2}(\Omega)\right]^{2 \times 2} .
$$

We now define $H_{0}:=\left\{\boldsymbol{\tau} \in H: \quad \int_{\Omega} \operatorname{tr}(\boldsymbol{\tau})=0\right\}$ and note that $H=H_{0} \oplus \mathbb{R} \mathbf{I}$, that is for any $\boldsymbol{\tau} \in H$ there exist unique $\boldsymbol{\tau}_{0} \in H_{0}$ and $d:=\frac{1}{2|\Omega|} \int_{\Omega} \operatorname{tr}(\boldsymbol{\tau}) \in \mathbb{R}$ such that $\boldsymbol{\tau}=\boldsymbol{\tau}_{0}+d \mathbf{I}$. According to this decomposition and the identities given in (2.7), we find that (2.4) is equivalent to: find $(\boldsymbol{\sigma},(\mathbf{u}, \gamma)) \in H_{0} \times Q$ such that

$$
\begin{aligned}
a(\boldsymbol{\sigma}, \boldsymbol{\tau})+b(\boldsymbol{\tau},(\mathbf{u}, \boldsymbol{\gamma})) & =0 \quad \forall \boldsymbol{\tau} \in H_{0}, \\
b(\boldsymbol{\sigma},(\mathbf{v}, \boldsymbol{\eta})) & =-\int_{\Omega} \mathbf{f} \cdot \mathbf{v} \quad \forall(\mathbf{v}, \boldsymbol{\eta}) \in Q .
\end{aligned}
$$

Indeed, taking $\boldsymbol{\tau}=\mathbf{I}$ in the first equation of (2.4) and using (2.7) we deduce that $\boldsymbol{\sigma} \in H_{0}$, whence one can show that $(\boldsymbol{\sigma},(\mathbf{u}, \boldsymbol{\gamma})) \in H \times Q$ is solution of (2.4) if and only if $\boldsymbol{\sigma} \in H_{0}$ and $(\boldsymbol{\sigma},(\mathbf{u}, \boldsymbol{\gamma}))$ is solution of (2.10).

We observe next that (2.10) satisfies the hypotheses of the Babuška-Brezzi theory (see [10] or [1]), which yields the unique solvability and continuous dependence of this variational formulation. For this purpose we need the following lemma, which will also be used later on. 
Lemma 2.1. There exists $c_{1}>0$, depending only on $\Omega$, such that

$$
c_{1}\|\boldsymbol{\tau}\|_{\left[L^{2}(\Omega)\right]^{2 \times 2}}^{2} \leq\left\|\boldsymbol{\tau}^{\mathrm{d}}\right\|_{\left[L^{2}(\Omega)\right]^{2 \times 2}}^{2}+\|\operatorname{div}(\boldsymbol{\tau})\|_{\left[L^{2}(\Omega)\right]^{2}}^{2} \quad \forall \boldsymbol{\tau} \in H_{0}
$$

and

$$
c_{1}\left\|\boldsymbol{\tau}_{0}\right\|_{\left[L^{2}(\Omega)\right]^{2 \times 2}}^{2} \leq\left\|\boldsymbol{\tau}^{\mathrm{d}}\right\|_{\left[L^{2}(\Omega)\right]^{2 \times 2}}^{2}+\|\operatorname{div}(\boldsymbol{\tau})\|_{\left[L^{2}(\Omega)\right]^{2}}^{2} \quad \forall \boldsymbol{\tau} \in H .
$$

Proof. For the proof of (2.11) we refer to Lemma 3.1 in [3] or Proposition 3.1 of Chapter IV in [10]. Then, given $\boldsymbol{\tau}=\boldsymbol{\tau}_{0}+d \mathbf{I} \in H$, with $\boldsymbol{\tau}_{0} \in H_{0}$ and $d \in \mathbb{R}$, we note that (2.12) follows from (2.11) and the fact that $\boldsymbol{\tau}_{0}^{\mathrm{d}}=\boldsymbol{\tau}^{\mathrm{d}}$ and $\operatorname{div}\left(\boldsymbol{\tau}_{0}\right)=\operatorname{div}(\boldsymbol{\tau})$.

Now, we deduce from (2.6) that $V:=\left\{\boldsymbol{\tau} \in H_{0}: \boldsymbol{\tau}=\boldsymbol{\tau}^{\mathrm{t}}\right.$ and $\operatorname{div}(\boldsymbol{\tau})=0$ in $\left.\Omega\right\}$ is the null space of $b$, whence (2.9) and (2.11) imply $a(\boldsymbol{\tau}, \boldsymbol{\tau}) \geq \frac{c_{1}}{2 \mu}\|\boldsymbol{\tau}\|_{\left[L^{2}(\Omega)\right]^{2 \times 2}}^{2}$ for all $\boldsymbol{\tau} \in V$. This shows that $a$ is $V$-elliptic, independently of the Lamé constant $\lambda$. Similarly, it is easy to see from (2.5) that $a$ is bounded with a constant given by $\frac{1}{2 \mu}$. For a detailed proof of the continuous inf-sup condition satisfied by $b$ we refer to Lemma 4.3 in [6], while the boundedness of $b$ is clear from (2.6). Alternatively, the well posedness of (2.10) is also proved in [4].

\subsection{Mixed boundary conditions}

We now deal with the mixed boundary conditions, as set originally in (2.1). To this end we recall that $\left[H^{-1 / 2}\left(\Gamma_{N}\right)\right]^{2}$ is the dual of the space $\left[H_{00}^{1 / 2}\left(\Gamma_{N}\right)\right]^{2}:=\left\{\left.\mathbf{v}\right|_{\Gamma_{N}}: \mathbf{v} \in\left[H^{1}(\Omega)\right]^{2}, \mathbf{v}=0\right.$ on $\left.\Gamma_{D}\right\}$, and that the associated duality pairing with respect to the $\left[L^{2}\left(\Gamma_{N}\right)\right]^{2}$-inner product is denoted by $\langle\cdot, \cdot\rangle_{\Gamma_{N}}$. Hence, we follow the approach from [5] and impose the Neumann condition in a weak sense, which yields the introduction of a Lagrange multiplier $\boldsymbol{\xi}:=-\left.\mathbf{u}\right|_{\Gamma_{N}} \in\left[H_{00}^{1 / 2}\left(\Gamma_{N}\right)\right]^{2}$ as an auxiliary unknown. In this way, proceeding with the remaining terms as in the previous case, we arrive to the following dual-mixed variational formulation of $(2.1)-(2.2)$ : find $(\boldsymbol{\sigma},(\mathbf{u}, \boldsymbol{\gamma}, \boldsymbol{\xi})) \in H \times \tilde{Q}$ such that

$$
\begin{aligned}
a(\boldsymbol{\sigma}, \boldsymbol{\tau})+\tilde{b}(\boldsymbol{\tau},(\mathbf{u}, \boldsymbol{\gamma}, \boldsymbol{\xi})) & =0 \quad \forall \boldsymbol{\tau} \in H, \\
\tilde{b}(\boldsymbol{\sigma},(\mathbf{v}, \boldsymbol{\eta}, \boldsymbol{\chi})) & =-\int_{\Omega} \mathbf{f} \cdot \mathbf{v}+\langle\mathbf{g}, \boldsymbol{\chi}\rangle_{\Gamma_{N}} \quad \forall(\mathbf{v}, \boldsymbol{\eta}, \boldsymbol{\chi}) \in \tilde{Q},
\end{aligned}
$$

where $\tilde{Q}:=\left[L^{2}(\Omega)\right]^{2} \times\left[L^{2}(\Omega)\right]_{\text {skew }}^{2 \times 2} \times\left[H_{00}^{1 / 2}\left(\Gamma_{N}\right)\right]^{2}$ and the bilinear form $\tilde{b}: H \times \tilde{Q} \rightarrow \mathbb{R}$ arises after adding the Neumann boundary term to $b$, that is

$$
\tilde{b}(\boldsymbol{\tau},(\mathbf{v}, \boldsymbol{\eta}, \boldsymbol{\chi})):=\int_{\Omega} \mathbf{v} \cdot \operatorname{div}(\boldsymbol{\tau})+\int_{\Omega} \boldsymbol{\tau}: \boldsymbol{\eta}+\langle\boldsymbol{\tau} \boldsymbol{\nu}, \boldsymbol{\chi}\rangle_{\Gamma_{N}}
$$

At this point we find it important to remark that, differently from (2.10), the formulation (2.13) can not be set equivalently in $H_{0} \times \tilde{Q}$. Indeed, from the relation $\boldsymbol{\sigma}=\mathcal{C} \mathbf{e}(\mathbf{u})$ we observe that $\operatorname{tr}(\boldsymbol{\sigma})=2(\lambda+\mu) \operatorname{tr}(\mathbf{e}(\mathbf{u}))=$ $2(\lambda+\mu) \operatorname{div}(\mathbf{u})$, and hence, using that $\mathbf{u}=0$ on $\Gamma_{D}$, we deduce that $\int_{\Omega} \operatorname{tr}(\boldsymbol{\sigma})=2(\lambda+\mu) \int_{\Omega} \operatorname{div}(\mathbf{u})=$ $2(\lambda+\mu) \int_{\Gamma_{N}} \mathbf{u} \cdot \boldsymbol{\nu}$. The above shows that the presence of the Neumann boundary condition on $\Gamma_{N}$ does not allow to guarantee that the stress $\boldsymbol{\sigma}$ belongs to $H_{0}$, and hence inequality (2.11) can not be applied in this case. Nevertheless, we prove below that this difficulty can be circumvented by combining (2.12) with the equivalence result given by the following lemma.

Lemma 2.2. There exists $c_{2}>0$, depending only on $\Gamma_{N}$ and $\Omega$, such that

$$
c_{2}\|\boldsymbol{\tau}\|_{H(\operatorname{div} ; \Omega)}^{2} \leq\left\|\boldsymbol{\tau}_{0}\right\|_{H(\operatorname{div} ; \Omega)}^{2} \quad \forall \boldsymbol{\tau} \in H \quad \text { such that } \quad \boldsymbol{\tau} \boldsymbol{\nu}=0 \quad \text { on } \quad \Gamma_{N} .
$$


Proof. Given $\boldsymbol{\tau}=\boldsymbol{\tau}_{0}+d \mathbf{I} \in H$, with $\boldsymbol{\tau}_{0} \in H_{0}$ and $d \in \mathbb{R}$, and such that $\boldsymbol{\tau} \boldsymbol{\nu}=0$ on $\Gamma_{N}$, we note that $d \boldsymbol{\nu}=-\boldsymbol{\tau}_{0} \boldsymbol{\nu}$ on $\Gamma_{N}$, and hence, with a constant $\tilde{c}_{2}>0$, depending on $\Gamma_{N}$,

$$
|d|\|\boldsymbol{\nu}\|_{\left[H^{-1 / 2}\left(\Gamma_{N}\right)\right]^{2}}=\left\|\boldsymbol{\tau}_{0} \boldsymbol{\nu}\right\|_{\left[H^{-1 / 2}\left(\Gamma_{N}\right)\right]^{2}} \leq \tilde{c}_{2}\left\|\boldsymbol{\tau}_{0} \boldsymbol{\nu}\right\|_{\left[H^{-1 / 2}(\Gamma)\right]^{2}} \leq \tilde{c}_{2}\left\|\boldsymbol{\tau}_{0}\right\|_{H(\operatorname{div} ; \Omega)}
$$

which yields $|d| \leq \frac{\tilde{c}_{2}}{\|\boldsymbol{\nu}\|_{\left[H^{-1 / 2}\left(\Gamma_{N}\right)\right]^{2}}}\left\|\boldsymbol{\tau}_{0}\right\|_{H(\mathbf{d i v} ; \Omega)}$.

This inequality and the fact that $\|\boldsymbol{\tau}\|_{H(\operatorname{div} ; \Omega)}^{2}=\left\|\boldsymbol{\tau}_{0}\right\|_{H(\operatorname{div} ; \Omega)}^{2}+2 d^{2}|\Omega| \operatorname{imply}(2.15)$.

Similarly to (2.10), we now check that (2.13) satisfies the hypotheses of the Babuška-Brezzi theory. Indeed, we first deduce from (2.14) that the null space of the bilinear form $\tilde{b}$ is given by $\tilde{V}:=\{\boldsymbol{\tau} \in H:$ $\boldsymbol{\tau}=\boldsymbol{\tau}^{\mathrm{t}}$ and $\operatorname{div}(\boldsymbol{\tau})=0$ in $\Omega$, and $\boldsymbol{\tau} \boldsymbol{\nu}=0$ on $\left.\Gamma_{N}\right\}$. Thus, combining (2.9), (2.12), and (2.15), we find that $a(\boldsymbol{\tau}, \boldsymbol{\tau}) \geq \frac{c_{1} c_{2}}{2 \mu}\|\boldsymbol{\tau}\|_{\left[L^{2}(\Omega)\right]^{2 \times 2}}^{2}$ for all $\boldsymbol{\tau} \in \tilde{V}$, which shows that $a$ is $\tilde{V}$-elliptic, independently of the Lamé constant $\lambda$. Next, the boundedness of $\tilde{b}$ is straightforward and the proof of the continuous inf-sup condition for $\tilde{b}$ reduces to combine the corresponding proof for $b$ (see Lem. 4.3 in [6]) with the following result.

Lemma 2.3. Let $\tilde{H}:=\left\{\boldsymbol{\tau} \in H: \boldsymbol{\tau}=\boldsymbol{\tau}^{\mathrm{t}}\right.$ and $\operatorname{div}(\boldsymbol{\tau})=0$ in $\left.\Omega\right\}$. Then there exists $\tilde{\beta}>0$, depending only on $\Gamma_{D}$ and $\Omega$, such that

$$
\sup _{\substack{\boldsymbol{\tau} \in \tilde{H} \\ \boldsymbol{\tau} \neq 0}} \frac{\langle\boldsymbol{\tau} \boldsymbol{\nu}, \boldsymbol{\chi}\rangle_{\Gamma_{N}}}{\|\boldsymbol{\tau}\|_{H(\operatorname{div} ; \Omega)}} \geq \tilde{\beta}\|\chi\|_{\left[H_{00}^{1 / 2}\left(\Gamma_{N}\right)\right]^{2}} \quad \forall \boldsymbol{\chi} \in\left[H_{00}^{1 / 2}\left(\Gamma_{N}\right)\right]^{2}
$$

Proof. Given $\boldsymbol{\varphi} \in\left[H^{-1 / 2}\left(\Gamma_{N}\right)\right]^{2}$, we let $\boldsymbol{\tau}(\boldsymbol{\varphi}):=\mathbf{e}(\mathbf{z})$ where $\mathbf{z} \in\left[H_{\Gamma_{D}}^{1}(\Omega)\right]^{2}$ is the weak solution of the boundary value problem:

$$
\operatorname{div} \mathbf{e}(\mathbf{z})=0 \quad \text { in } \Omega, \quad \mathbf{z}=0 \quad \text { on } \quad \Gamma_{D}, \quad \mathbf{e}(\mathbf{z}) \boldsymbol{\nu}=\boldsymbol{\varphi} \quad \text { on } \quad \Gamma_{N}
$$

The unique solvability of (2.17) follows from Korn's second inequality (see Chap. VI in [8]) and Lax-Milgram Lemma. We observe that $\boldsymbol{\tau}(\boldsymbol{\varphi}) \in \tilde{H}$ and $\boldsymbol{\tau}(\boldsymbol{\varphi}) \boldsymbol{\nu}=\boldsymbol{\varphi}$ on $\Gamma_{N}$. In addition, applying the continuous dependence result for (2.17), we find that there exists $C>0$, depending only on $\Gamma_{D}$ and $\Omega$, such that $\|\boldsymbol{\tau}(\boldsymbol{\varphi})\|_{H(\operatorname{div} ; \Omega)}=$ $\|\mathbf{e}(\mathbf{z})\|_{\left[L^{2}(\Omega)\right]^{2 \times 2}} \leq\|\mathbf{z}\|_{\left[H^{1}(\Omega)\right]^{2}} \leq C\|\boldsymbol{\varphi}\|_{\left[H^{-1 / 2}\left(\Gamma_{N}\right)\right]^{2}}$. Hence, for each $\chi \in\left[H_{00}^{1 / 2}\left(\Gamma_{N}\right)\right]^{2}$ we can write

$$
\sup _{\substack{\boldsymbol{\tau} \in \tilde{H} \\ \boldsymbol{\tau} \neq 0}} \frac{\left|\langle\boldsymbol{\tau} \boldsymbol{\nu}, \boldsymbol{\chi}\rangle_{\Gamma_{N}}\right|}{\|\boldsymbol{\tau}\|_{H(\operatorname{div} ; \Omega)}} \geq \frac{\left|\langle\boldsymbol{\tau}(\boldsymbol{\varphi}) \boldsymbol{\nu}, \boldsymbol{\chi}\rangle_{\Gamma_{N}}\right|}{\|\boldsymbol{\tau}(\boldsymbol{\varphi})\|_{H(\operatorname{div} ; \Omega)}} \geq \frac{1}{C} \frac{\left|\langle\boldsymbol{\varphi}, \boldsymbol{\chi}\rangle_{\Gamma_{N}}\right|}{\|\boldsymbol{\varphi}\|_{\left[H^{-1 / 2}\left(\Gamma_{N}\right)\right]^{2}}} \quad \forall \boldsymbol{\varphi} \in\left[H^{-1 / 2}\left(\Gamma_{N}\right)\right]^{2}
$$

which, according to a classical result in functional analysis, yields $(2.16)$ with $\tilde{\beta}=\frac{1}{C}$.

We end this section by recalling that the main difficulty in applying the Galerkin schemes associated with (2.10) and (2.13) is the derivation of explicit finite element subspaces on which the ellipticity of $a$ and the discrete inf-sup conditions of $b$ and $\tilde{b}$ hold. As mentioned in Section 1, several approximations, at least for the case of pure Dirichlet boundary conditions, are already available in the literature (see, e.g. $[1,10,12,13,32]$ ), which, however, are not always satisfactory since they either are rather expensive (because of the number of degrees of freedom involved) or yield higher polynomial degrees than expected. Moreover, up to the author's knowledge, no much has been done for mixed boundary conditions with non-homogeneous Neumann data. The PEERS elements are certainly applicable to this case, but since the Neumann boundary condition becomes now essential, it needs to be approximated for the setting of the discrete system, which yields a non-conforming Galerkin scheme. These facts have usually motivated the need of alternative methods, such as the augmented variational formulations to be proposed in the next section. 


\section{The AUgmented DUAL-MIXED VARIATIONAL FORMUlations}

\subsection{Dirichlet boundary conditions}

We suggest to enrich the corresponding dual-mixed variational formulation (2.10) with residuals arising from the constitutive and equilibrium equations, and from the relation defining the rotation as a function of the displacement. More precisely, we substract the second from the first equation of (2.10) and then add the Galerkin least-squares terms given by

$$
\begin{gathered}
\kappa_{1} \int_{\Omega}\left(\mathbf{e}(\mathbf{u})-\mathcal{C}^{-1} \boldsymbol{\sigma}\right):\left(\mathbf{e}(\mathbf{v})+\mathcal{C}^{-1} \boldsymbol{\tau}\right)=0, \\
\kappa_{2} \int_{\Omega} \operatorname{div}(\boldsymbol{\sigma}) \cdot \operatorname{div}(\boldsymbol{\tau})=-\kappa_{2} \int_{\Omega} \mathbf{f} \cdot \operatorname{div}(\boldsymbol{\tau}),
\end{gathered}
$$

and

$$
\kappa_{3} \int_{\Omega}\left(\boldsymbol{\gamma}-\frac{1}{2}\left(\nabla \mathbf{u}-(\nabla \mathbf{u})^{\mathrm{t}}\right)\right):\left(\boldsymbol{\eta}+\frac{1}{2}\left(\nabla \mathbf{v}-(\nabla \mathbf{v})^{\mathrm{t}}\right)\right)=0,
$$

for all $(\boldsymbol{\tau}, \mathbf{v}, \boldsymbol{\eta}) \in H_{0} \times\left[H_{0}^{1}(\Omega)\right]^{2} \times\left[L^{2}(\Omega)\right]_{\text {skew }}^{2 \times 2}$, where $\left(\kappa_{1}, \kappa_{2}, \kappa_{3}\right)$ is a vector of positive parameters to be specified later. We just remark in advance that we will be able to chose them independently of the Lamé constant $\lambda$. We also notice here that (3.1) and (3.3) implicitly require now the displacement $\mathbf{u}$ to live in the smaller space $\left[H_{0}^{1}(\Omega)\right]^{2}$.

In this way, instead of $(2.10)$ we propose the following augmented variational formulation: find $(\boldsymbol{\sigma}, \mathbf{u}, \boldsymbol{\gamma}) \in$ $\mathbf{H}_{0}:=H_{0} \times\left[H_{0}^{1}(\Omega)\right]^{2} \times\left[L^{2}(\Omega)\right]_{\text {skew }}^{2 \times 2}$ such that

$$
A((\boldsymbol{\sigma}, \mathbf{u}, \boldsymbol{\gamma}),(\boldsymbol{\tau}, \mathbf{v}, \boldsymbol{\eta}))=F(\boldsymbol{\tau}, \mathbf{v}, \boldsymbol{\eta}) \quad \forall(\boldsymbol{\tau}, \mathbf{v}, \boldsymbol{\eta}) \in \mathbf{H}_{0}
$$

where the bilinear form $A: \mathbf{H}_{0} \times \mathbf{H}_{0} \rightarrow \mathbb{R}$ and the functional $F: \mathbf{H}_{0} \rightarrow \mathbb{R}$ are defined by

$$
\begin{aligned}
& A((\boldsymbol{\sigma}, \mathbf{u}, \boldsymbol{\gamma}),(\boldsymbol{\tau}, \mathbf{v}, \boldsymbol{\eta})):=\int_{\Omega} \mathcal{C}^{-1} \boldsymbol{\sigma}: \boldsymbol{\tau}+\int_{\Omega} \mathbf{u} \cdot \operatorname{div}(\boldsymbol{\tau})+\int_{\Omega} \boldsymbol{\gamma}: \boldsymbol{\tau}-\int_{\Omega} \mathbf{v} \cdot \operatorname{div}(\boldsymbol{\sigma})-\int_{\Omega} \boldsymbol{\eta}: \boldsymbol{\sigma} \\
& +\kappa_{1} \int_{\Omega}\left(\mathbf{e}(\mathbf{u})-\mathcal{C}^{-1} \boldsymbol{\sigma}\right):\left(\mathbf{e}(\mathbf{v})+\mathcal{C}^{-1} \boldsymbol{\tau}\right)+\kappa_{2} \int_{\Omega} \operatorname{div}(\boldsymbol{\sigma}) \cdot \operatorname{div}(\boldsymbol{\tau}) \\
& +\kappa_{3} \int_{\Omega}\left(\boldsymbol{\gamma}-\frac{1}{2}\left(\nabla \mathbf{u}-(\nabla \mathbf{u})^{\mathrm{t}}\right)\right):\left(\boldsymbol{\eta}+\frac{1}{2}\left(\nabla \mathbf{v}-(\nabla \mathbf{v})^{\mathrm{t}}\right)\right)
\end{aligned}
$$

and

$$
F(\boldsymbol{\tau}, \mathbf{v}, \boldsymbol{\eta}):=\int_{\Omega} \mathbf{f} \cdot\left(\mathbf{v}-\kappa_{2} \operatorname{div}(\boldsymbol{\tau})\right) .
$$

The idea is to choose $\left(\kappa_{1}, \kappa_{2}, \kappa_{3}\right)$ so that $A$ becomes strongly coercive and bounded on $\mathbf{H}_{0}$, with constants independent of $\lambda$, with respect to the norm $\|\cdot\|_{\mathbf{H}_{0}}$ defined by

$$
\|(\boldsymbol{\tau}, \mathbf{v}, \boldsymbol{\eta})\|_{\mathbf{H}_{0}}:=\left\{\|\boldsymbol{\tau}\|_{H(\mathbf{d i v} ; \Omega)}^{2}+|\mathbf{v}|_{\left[H^{1}(\Omega)\right]^{2}}^{2}+\|\boldsymbol{\eta}\|_{\left[L^{2}(\Omega)\right]^{2 \times 2}}^{2}\right\}^{1 / 2} \quad \forall(\boldsymbol{\tau}, \mathbf{v}, \boldsymbol{\eta}) \in \mathbf{H}_{0}
$$

We first notice, after simple computations, that

$$
\int_{\Omega}\left(\mathbf{e}(\mathbf{v})-\mathcal{C}^{-1} \boldsymbol{\tau}\right):\left(\mathbf{e}(\mathbf{v})+\mathcal{C}^{-1} \boldsymbol{\tau}\right)=\|\mathbf{e}(\mathbf{v})\|_{\left[L^{2}(\Omega)\right]^{2 \times 2}}^{2}-\left\|\mathcal{C}^{-1} \boldsymbol{\tau}\right\|_{\left[L^{2}(\Omega)\right]^{2 \times 2}}^{2},
$$


and that

$$
\begin{aligned}
\int_{\Omega}\left(\boldsymbol{\eta}-\frac{1}{2}\left(\nabla \mathbf{v}-(\nabla \mathbf{v})^{\mathrm{t}}\right)\right):\left(\boldsymbol{\eta}+\frac{1}{2}\left(\nabla \mathbf{v}-(\nabla \mathbf{v})^{\mathrm{t}}\right)\right) \\
=\|\boldsymbol{\eta}\|_{\left[L^{2}(\Omega)\right]^{2 \times 2}}^{2}+\|\mathbf{e}(\mathbf{v})\|_{\left[L^{2}(\Omega)\right]^{2 \times 2}}^{2}-|\mathbf{v}|_{\left[H^{1}(\Omega)\right]^{2}}^{2}
\end{aligned}
$$

which gives

$$
\begin{gathered}
A((\boldsymbol{\tau}, \mathbf{v}, \boldsymbol{\eta}),(\boldsymbol{\tau}, \mathbf{v}, \boldsymbol{\eta}))=\int_{\Omega} \mathcal{C}^{-1} \boldsymbol{\tau}: \boldsymbol{\tau}-\kappa_{1}\left\|\mathcal{C}^{-1} \boldsymbol{\tau}\right\|_{\left[L^{2}(\Omega)\right]^{2 \times 2}}^{2}+\left(\kappa_{1}+\kappa_{3}\right)\|\mathbf{e}(\mathbf{v})\|_{\left[L^{2}(\Omega)\right]^{2 \times 2}}^{2} \\
+\kappa_{2}\|\operatorname{div}(\boldsymbol{\tau})\|_{\left[L^{2}(\Omega)\right]^{2}}^{2}+\kappa_{3}\|\boldsymbol{\eta}\|_{\left[L^{2}(\Omega)\right]^{2 \times 2}}^{2}-\kappa_{3}|\mathbf{v}|_{\left[H^{1}(\Omega)\right]^{2}}^{2} .
\end{gathered}
$$

Next, using (2.5) and the inverse relation (2.3), and performing some algebraic manipulations, we find that

$$
\begin{gathered}
\int_{\Omega} \mathcal{C}^{-1} \boldsymbol{\tau}: \boldsymbol{\tau}-\kappa_{1}\left\|\mathcal{C}^{-1} \boldsymbol{\tau}\right\|_{\left[L^{2}(\Omega)\right]^{2 \times 2}}^{2}=\frac{1}{4 \mu}\left\{2\|\boldsymbol{\tau}\|_{\left[L^{2}(\Omega)\right]^{2 \times 2}}^{2}-\left(\frac{\lambda}{\lambda+\mu}\right) \int_{\Omega} \operatorname{tr}^{2}(\boldsymbol{\tau})\right\} \\
-\frac{\kappa_{1}}{8 \mu^{2}}\left\{2\|\boldsymbol{\tau}\|_{\left[L^{2}(\Omega)\right]^{2 \times 2}}^{2}-2\left(\frac{\lambda}{\lambda+\mu}\right) \int_{\Omega} \operatorname{tr}^{2}(\boldsymbol{\tau})+\left(\frac{\lambda}{\lambda+\mu}\right)^{2} \int_{\Omega} \operatorname{tr}^{2}(\boldsymbol{\tau})\right\} \\
=\frac{1}{2 \mu}\left(1-\frac{\kappa_{1}}{2 \mu}\right)\left\|\boldsymbol{\tau}^{\mathrm{d}}\right\|_{\left[L^{2}(\Omega)\right]^{2 \times 2}}^{2}+\frac{1}{4(\lambda+\mu)}\left(1-\frac{\kappa_{1}}{2(\lambda+\mu)}\right) \int_{\Omega} \operatorname{tr}^{2}(\boldsymbol{\tau}),
\end{gathered}
$$

from which we observe that it suffices to choose $\kappa_{1}$ so that $1-\frac{\kappa_{1}}{2(\lambda+\mu)} \geq 0$ and $1-\frac{\kappa_{1}}{2 \mu}>0$, that is $\quad 0<\kappa_{1}<2 \mu$, which yields

$$
\int_{\Omega} \mathcal{C}^{-1} \boldsymbol{\tau}: \boldsymbol{\tau}-\kappa_{1}\left\|\mathcal{C}^{-1} \boldsymbol{\tau}\right\|_{\left[L^{2}(\Omega)\right]^{2 \times 2}}^{2} \geq \frac{1}{2 \mu}\left(1-\frac{\kappa_{1}}{2 \mu}\right)\left\|\boldsymbol{\tau}^{\mathrm{d}}\right\|_{\left[L^{2}(\Omega)\right]^{2 \times 2}}^{2} \quad \forall \boldsymbol{\tau} \in H .
$$

In this way, (3.8), (3.10), and (2.11) (cf. Lem. 2.1) imply that

$$
\begin{gathered}
A((\boldsymbol{\tau}, \mathbf{v}, \boldsymbol{\eta}),(\boldsymbol{\tau}, \mathbf{v}, \boldsymbol{\eta})) \geq \alpha_{2}\|\boldsymbol{\tau}\|_{H(\operatorname{div} ; \Omega)}^{2}+\left(\kappa_{1}+\kappa_{3}\right)\|\mathbf{e}(\mathbf{v})\|_{\left[L^{2}(\Omega)\right]^{2 \times 2}}^{2} \\
+\kappa_{3}\|\boldsymbol{\eta}\|_{\left[L^{2}(\Omega)\right]^{2 \times 2}}^{2}-\kappa_{3}|\mathbf{v}|_{\left[H^{1}(\Omega)\right]^{2}}^{2} \quad \forall(\boldsymbol{\tau}, \mathbf{v}, \boldsymbol{\eta}) \in \mathbf{H}_{0},
\end{gathered}
$$

where

$$
\alpha_{2}:=\min \left\{\alpha_{1} c_{1}, \frac{\kappa_{2}}{2}\right\} \quad \text { and } \quad \alpha_{1}:=\min \left\{\frac{1}{2 \mu}\left(1-\frac{\kappa_{1}}{2 \mu}\right), \frac{\kappa_{2}}{2}\right\} .
$$

But, Korn's first inequality (see, e.g. Thm. 10.1 in [28] or equation (2.11) in [23]) establishes that

$$
\|\mathbf{e}(\mathbf{v})\|_{\left[L^{2}(\Omega)\right]^{2 \times 2}}^{2} \geq \frac{1}{2}|v|_{\left[H^{1}(\Omega)\right]^{2}}^{2} \quad \forall v \in\left[H_{0}^{1}(\Omega)\right]^{2},
$$

and hence (3.11) becomes

$$
A((\boldsymbol{\tau}, \mathbf{v}, \boldsymbol{\eta}),(\boldsymbol{\tau}, \mathbf{v}, \boldsymbol{\eta})) \geq \alpha_{2}\|\boldsymbol{\tau}\|_{H(\operatorname{div} ; \Omega)}^{2}+\frac{\left(\kappa_{1}-\kappa_{3}\right)}{2}|\mathbf{v}|_{\left[H^{1}(\Omega)\right]^{2}}^{2}+\kappa_{3}\|\boldsymbol{\eta}\|_{\left[L^{2}(\Omega)\right]^{2 \times 2}}^{2}
$$

for all $(\boldsymbol{\tau}, \mathbf{v}, \boldsymbol{\eta}) \in \mathbf{H}_{0}$, from which we deduce that it suffices to take $0<\kappa_{3}<\kappa_{1}$ to obtain the strong coerciveness of $A$ on the space $\mathbf{H}_{0}$.

Now, as we notice from the above estimates, there is no further restriction on $\kappa_{2}$ besides being positive. For instance, as suggested by the definition of $\alpha_{1}$, we may choose $\kappa_{2}=\frac{1}{\mu}\left(1-\frac{\kappa_{1}}{2 \mu}\right)$ so that $\alpha_{1}=\frac{\kappa_{2}}{2}$ and 
hence $\alpha_{2}=\frac{\kappa_{2}}{2} \min \left\{c_{1}, 1\right\}$. Therefore, according to the definition of $A$ (cf. (3.5)), and taking into account that the constants appearing in the inverse tensor $\mathcal{C}^{-1}$ are of $O(1 / \mu)(c f .(2.3))$, it becomes suitable to consider $\kappa_{1}=C_{1} \mu$ and $\kappa_{3}=C_{3} \kappa_{1}$, with any $\left.C_{1} \in\right] 0,2\left[\right.$ and any $\left.C_{3} \in\right] 0,1[$. In this way, the coerciveness constant of $A$ arising from (3.14) depends only on $\mu, \frac{1}{\mu}$, and $c_{1}$. In particular, the feasible choice $C_{1}=1$ and $C_{3}=\frac{1}{2}$ yields

$$
\kappa_{1}=\mu, \quad \kappa_{2}=\frac{1}{2 \mu}, \quad \kappa_{3}=\frac{\mu}{2}, \quad \alpha_{1}=\frac{1}{4 \mu}, \quad \text { and } \quad \alpha_{2}=\frac{1}{4 \mu} \min \left\{c_{1}, 1\right\},
$$

whereas $C_{1}=\frac{3}{2}$ and $C_{3}=\frac{2}{3}$ give

$$
\kappa_{1}=\frac{3 \mu}{2}, \quad \kappa_{2}=\frac{1}{4 \mu}, \quad \kappa_{3}=\mu, \quad \alpha_{1}=\frac{1}{8 \mu}, \quad \text { and } \quad \alpha_{2}=\frac{1}{8 \mu} \min \left\{c_{1}, 1\right\} .
$$

On the other hand, it is easy to see from (2.3) that $\left\|\mathcal{C}^{-1} \boldsymbol{\tau}\right\|_{\left[L^{2}(\Omega)\right]^{2 \times 2}} \leq \frac{1}{2 \mu}\|\boldsymbol{\tau}\|_{\left[L^{2}(\Omega)\right]^{2 \times 2}}$ for all $\boldsymbol{\tau} \in\left[L^{2}(\Omega)\right]^{2 \times 2}$. Hence, applying Cauchy-Schwarz's inequality to each term on the right hand side of (3.5) we conclude that $A$ is bounded with a constant depending only on $\mu, \kappa_{1}, \kappa_{2}$, and $\kappa_{3}$.

We have thus proved the following main result.

Theorem 3.1. Assume that $\left(\kappa_{1}, \kappa_{2}, \kappa_{3}\right)$ is independent of $\lambda$ and such that $0<\kappa_{1}<2 \mu, 0<\kappa_{2}$, and $0<\kappa_{3}<\kappa_{1}$. Then, there exist positive constants $M, \alpha$, independent of $\lambda$, such that

$$
|A((\boldsymbol{\sigma}, \mathbf{u}, \boldsymbol{\gamma}),(\boldsymbol{\tau}, \mathbf{v}, \boldsymbol{\eta}))| \leq M\|(\boldsymbol{\sigma}, \mathbf{u}, \boldsymbol{\gamma})\|_{\mathbf{H}_{0}}\|(\boldsymbol{\tau}, \mathbf{v}, \boldsymbol{\eta})\|_{\mathbf{H}_{0}}
$$

and

$$
A((\boldsymbol{\tau}, \mathbf{v}, \boldsymbol{\eta}),(\boldsymbol{\tau}, \mathbf{v}, \boldsymbol{\eta})) \geq \alpha\|(\boldsymbol{\tau}, \mathbf{v}, \boldsymbol{\eta})\|_{\mathbf{H}_{0}}^{2}
$$

for all $(\boldsymbol{\sigma}, \mathbf{u}, \boldsymbol{\gamma}),(\boldsymbol{\tau}, \mathbf{v}, \boldsymbol{\eta}) \in \mathbf{H}_{0}$. In particular, taking $\kappa_{1}=C_{1} \mu, \kappa_{2}=\frac{1}{\mu}\left(1-\frac{\kappa_{1}}{2 \mu}\right)$, and $\kappa_{3}=C_{3} \kappa_{1}$, with any $\left.C_{1} \in\right] 0,2\left[\right.$ and any $\left.C_{3} \in\right] 0,1\left[\right.$, yields $M$ and $\alpha$ depending only on $\mu, \frac{1}{\mu}$, and $c_{1}$.

In addition, the well posedness of (3.4) is now easily established.

Theorem 3.2. Assume the same hypotheses of Theorem 3.1. Then the augmented variational formulation (3.4) has a unique solution $(\boldsymbol{\sigma}, \mathbf{u}, \boldsymbol{\gamma}) \in \mathbf{H}_{0}$, and there exists a positive constant $C$, independent of $\lambda$, such that $\|(\boldsymbol{\sigma}, \mathbf{u}, \gamma)\|_{\mathbf{H}_{0}} \leq C\|F\| \leq C\|\mathbf{f}\|_{\left[L^{2}(\Omega)\right]^{2}}$.

Proof. The linear functional $F$ (see (3.6)) is continuous with a norm bounded above by $\left(1+\kappa_{2}\right)\|\mathbf{f}\|_{\left[L^{2}(\Omega)\right]^{2}}$. Therefore, the present proof is a simple consequence of Theorem 3.1 and the well known Lax-Milgram lemma.

\subsection{Mixed boundary conditions}

We now deal with mixed boundary conditions and suggest to enrich the corresponding dual-mixed variational formulation (2.13) with the same Galerkin least-squares terms employed in the previous case (see (3.1), (3.2), and (3.3)). Consequently, instead of (2.13) we propose the following augmented variational formulation: find $((\boldsymbol{\sigma}, \mathbf{u}, \gamma), \boldsymbol{\xi}) \in \mathbf{H} \times \mathbf{Q}$ such that

$$
\begin{aligned}
A((\boldsymbol{\sigma}, \mathbf{u}, \boldsymbol{\gamma}),(\boldsymbol{\tau}, \mathbf{v}, \boldsymbol{\eta}))+B((\boldsymbol{\tau}, \mathbf{v}, \boldsymbol{\eta}), \boldsymbol{\xi}) & =F(\boldsymbol{\tau}, \mathbf{v}, \boldsymbol{\eta}) \quad \forall(\boldsymbol{\tau}, \mathbf{v}, \boldsymbol{\eta}) \in \mathbf{H}, \\
B((\boldsymbol{\sigma}, \mathbf{u}, \boldsymbol{\gamma}), \boldsymbol{\chi}) & =G(\boldsymbol{\chi}) \quad \forall \boldsymbol{\chi} \in \mathbf{Q},
\end{aligned}
$$

where $\mathbf{H}:=H \times\left[H_{\Gamma_{D}}^{1}(\Omega)\right]^{2} \times\left[L^{2}(\Omega)\right]_{\text {skew }}^{2 \times 2}$, with $\left[H_{\Gamma_{D}}^{1}(\Omega)\right]^{2}:=\left\{\mathbf{v} \in\left[H^{1}(\Omega)\right]^{2}: \quad \mathbf{v}=0\right.$ on $\left.\Gamma_{D}\right\}, \mathbf{Q}:=$ $\left[H_{00}^{1 / 2}\left(\Gamma_{N}\right)\right]^{2}, F: \mathbf{H} \rightarrow \mathbb{R}$ is the linear functional given by (3.6), $G: \mathbf{Q} \rightarrow \mathbb{R}$ is the linear functional defined by 
$G(\boldsymbol{\chi}):=\langle\mathbf{g}, \boldsymbol{\chi}\rangle_{\Gamma_{N}}$, the bilinear form $A: \mathbf{H} \times \mathbf{H} \rightarrow \mathbb{R}$ is given by (3.5), and the bilinear form $B: \mathbf{H} \times \mathbf{Q} \rightarrow \mathbb{R}$ is defined by

$$
B((\boldsymbol{\tau}, \mathbf{v}, \boldsymbol{\eta}), \boldsymbol{\chi}):=\langle\boldsymbol{\tau} \boldsymbol{\nu}, \boldsymbol{\chi}\rangle_{\Gamma_{N}} \quad \forall((\boldsymbol{\tau}, \mathbf{v}, \boldsymbol{\eta}), \boldsymbol{\chi}) \in \mathbf{H} \times \mathbf{Q} .
$$

We remark that, differently from (3.4), the variational formulation (3.17) is written in a dual-mixed structure since we need the term dealing with the Neumann boundary condition to be kept separate (in the form of $B$ ).

The goal now is to choose $\left(\kappa_{1}, \kappa_{2}, \kappa_{3}\right)$, independent of $\lambda$, so that (3.17) satisfies the hypotheses of the Babuška-Brezzi theory. We realize first that the null space of $B$ is given by

$$
\mathbf{V}:=\left\{(\boldsymbol{\tau}, \mathbf{v}, \boldsymbol{\eta}) \in \mathbf{H}: \quad \boldsymbol{\tau} \boldsymbol{\nu}=0 \quad \text { on } \quad \Gamma_{N}\right\} .
$$

Then, we note from (3.8) and (3.10) that for $0<\kappa_{1}<2 \mu$ and for each $(\boldsymbol{\tau}, \mathbf{v}, \boldsymbol{\eta}) \in \mathbf{H}$ there holds

$$
\begin{aligned}
A((\boldsymbol{\tau}, \mathbf{v}, \boldsymbol{\eta}),(\boldsymbol{\tau}, \mathbf{v}, \boldsymbol{\eta})) \geq \frac{1}{2 \mu}\left(1-\frac{\kappa_{1}}{2 \mu}\right) & \left\|\boldsymbol{\tau}^{\mathrm{d}}\right\|_{\left[L^{2}(\Omega)\right]^{2 \times 2}}^{2}+\left(\kappa_{1}+\kappa_{3}\right)\|\mathbf{e}(\mathbf{v})\|_{\left[L^{2}(\Omega)\right]^{2 \times 2}}^{2} \\
& +\kappa_{2}\|\operatorname{div}(\boldsymbol{\tau})\|_{\left[L^{2}(\Omega)\right]^{2}}^{2}+\kappa_{3}\|\boldsymbol{\eta}\|_{\left[L^{2}(\Omega)\right]^{2 \times 2}}^{2}-\kappa_{3}|\mathbf{v}|_{\left[H^{1}(\Omega)\right]^{2}}^{2},
\end{aligned}
$$

which, according to (2.12) (cf. Lem. 2.1), (3.19), and (2.15) (cf. Lem. 2.2), leads to

$$
\begin{aligned}
A((\boldsymbol{\tau}, \mathbf{v}, \boldsymbol{\eta}),(\boldsymbol{\tau}, \mathbf{v}, \boldsymbol{\eta})) \geq \alpha_{2} c_{2}\|\boldsymbol{\tau}\|_{H(\mathbf{d i v} ; \Omega)}^{2}+\left(\kappa_{1}+\kappa_{3}\right)\|\mathbf{e}(\mathbf{v})\|_{\left[L^{2}(\Omega)\right]^{2 \times 2}}^{2} \\
+\kappa_{3}\|\boldsymbol{\eta}\|_{\left[L^{2}(\Omega)\right]^{2 \times 2}}^{2}-\kappa_{3}|\mathbf{v}|_{\left[H^{1}(\Omega)\right]^{2}}^{2} \quad \forall(\boldsymbol{\tau}, \mathbf{v}, \boldsymbol{\eta}) \in \mathbf{V},
\end{aligned}
$$

where $\alpha_{2}$ is the constant defined in (3.12). Again, it follows that there is no further restriction on $\kappa_{2}$ besides being positive. Next, Korn's first inequality establishes now the existence of $k_{D} \in(0,1)$, depending only on $\Omega$ and $\Gamma_{D}$, such that

and hence (3.21) yields

$$
\|\mathbf{e}(\mathbf{v})\|_{\left.\left[L^{2}(\Omega)\right]\right]^{2 \times 2}}^{2} \geq k_{D}|\mathbf{v}|_{\left[H^{1}(\Omega)\right]^{2}}^{2} \quad \forall \mathbf{v} \in\left[H_{\Gamma_{D}}^{1}(\Omega)\right]^{2},
$$

$$
A((\boldsymbol{\tau}, \mathbf{v}, \boldsymbol{\eta}),(\boldsymbol{\tau}, \mathbf{v}, \boldsymbol{\eta})) \geq \alpha_{2} c_{2}\|\boldsymbol{\tau}\|_{H(\operatorname{div} ; \Omega)}^{2}+\left(\kappa_{1} k_{D}-\kappa_{3}\left(1-k_{D}\right)\right)|\mathbf{v}|_{\left[H^{1}(\Omega)\right]^{2}}^{2}+\kappa_{3}\|\boldsymbol{\eta}\|_{\left[L^{2}(\Omega)\right]^{2 \times 2}}^{2},
$$

for all $(\boldsymbol{\tau}, \mathbf{v}, \boldsymbol{\eta}) \in \mathbf{V}$, from which we conclude that it suffices to choose $0<\kappa_{3}<\frac{\kappa_{1} k_{D}}{1-k_{D}}$ to obtain the strong coerciveness of $A$ on $\mathbf{V}$ with respect to $\|\cdot\|_{\mathbf{H}}$ (also defined by (3.7)). At this point we remark that, differently from (3.13), the constant $k_{D}$ in (3.22) is not known in general. However, as described in Section 5 of the review article [23], there is a large class of two-dimensional domains for which $k_{D}$ can be estimated explicitly in terms of geometric constants. The corresponding results include star-shaped and simply connected regions (see $[24,25])$.

On the other hand, it was already established that $A$ is bounded with a constant depending only on $\mu, \kappa_{1}$, $\kappa_{2}$, and $\kappa_{3}$, and it is easy to see that $B$ is bounded, as well. In addition, as proved by Lemma $2.3, B$ also satisfies the continuous inf-sup condition with the constant $\tilde{\beta}$, which is independent of $\mu$ and $\lambda$.

In this way, we are able to state the following main result.

Theorem 3.3. Assume that $\left(\kappa_{1}, \kappa_{2}, \kappa_{3}\right)$ is independent of $\lambda$ and such that $0<\kappa_{1}<2 \mu, 0<\kappa_{2}$, and $0<\kappa_{3}<\frac{\kappa_{1} k_{D}}{1-k_{D}}$. Then, there exist positive constants $M$, $\tilde{\alpha}$, independent of $\lambda$, such that

$$
|A((\boldsymbol{\sigma}, \mathbf{u}, \boldsymbol{\gamma}),(\boldsymbol{\tau}, \mathbf{v}, \boldsymbol{\eta}))| \leq M\|(\boldsymbol{\sigma}, \mathbf{u}, \boldsymbol{\gamma})\|_{\mathbf{H}}\|(\boldsymbol{\tau}, \mathbf{v}, \boldsymbol{\eta})\|_{\mathbf{H}} \quad \forall(\boldsymbol{\sigma}, \mathbf{u}, \boldsymbol{\gamma}),(\boldsymbol{\tau}, \mathbf{v}, \boldsymbol{\eta}) \in \mathbf{H},
$$

and

$$
A((\boldsymbol{\tau}, \mathbf{v}, \boldsymbol{\eta}),(\boldsymbol{\tau}, \mathbf{v}, \boldsymbol{\eta})) \geq \tilde{\alpha}\|(\boldsymbol{\tau}, \mathbf{v}, \boldsymbol{\eta})\|_{\mathbf{H}}^{2} \quad \forall(\boldsymbol{\tau}, \mathbf{v}, \boldsymbol{\eta}) \in \mathbf{V} .
$$

In particular, taking $\kappa_{1}=C_{1} \mu, \kappa_{2}=\frac{1}{\mu}\left(1-\frac{\kappa_{1}}{2 \mu}\right)$, and $\kappa_{3}=C_{3} \frac{\kappa_{1} k_{D}}{1-k_{D}}$, with any $\left.C_{1} \in\right] 0,2\left[\right.$ and any $\left.C_{3} \in\right] 0,1[$, yields $M$ and $\tilde{\alpha}$ depending only on $\mu, \frac{1}{\mu}, c_{1}, c_{2}$, and $k_{D}$. On the other hand, there exist positive constants $\beta$ 
and $\tilde{\beta}$, independent of $\mu$ and $\lambda$, such that

$$
|B((\boldsymbol{\tau}, \mathbf{v}, \boldsymbol{\eta}), \boldsymbol{\chi})| \leq \beta\|(\boldsymbol{\tau}, \mathbf{v}, \boldsymbol{\eta})\|_{\mathbf{H}}\|\boldsymbol{\chi}\|_{\mathbf{Q}} \quad \forall((\boldsymbol{\tau}, \mathbf{v}, \boldsymbol{\eta}), \boldsymbol{\chi}) \in \mathbf{H} \times \mathbf{Q},
$$

and

$$
\sup _{\substack{(\boldsymbol{\tau}, \mathbf{v}, \boldsymbol{\eta}) \in \mathbf{H} \\(\boldsymbol{\tau}, \mathbf{v}, \boldsymbol{\eta}) \neq 0}} \frac{B((\boldsymbol{\tau}, \mathbf{v}, \boldsymbol{\eta}), \boldsymbol{\chi})}{\|(\boldsymbol{\tau}, \mathbf{v}, \boldsymbol{\eta})\|_{\mathbf{H}}} \geq \tilde{\beta}\|\chi\|_{\mathbf{Q}} \quad \forall \boldsymbol{\chi} \in \mathbf{Q} .
$$

The well-posedness of (3.17) can now be established.

Theorem 3.4. Assume the same hypotheses of Theorem 3.3. Then the augmented variational formulation (3.17) has a unique solution $((\boldsymbol{\sigma}, \mathbf{u}, \boldsymbol{\gamma}), \boldsymbol{\xi}) \in \mathbf{H} \times \mathbf{Q}$, and there exists a positive constant $C$, independent of $\lambda$, such that

$$
\|((\boldsymbol{\sigma}, \mathbf{u}, \boldsymbol{\gamma}), \boldsymbol{\xi})\|_{\mathbf{H} \times \mathbf{Q}} \leq C\{\|F\|+\|G\|\} \leq C\left\{\|\mathbf{f}\|_{\left[L^{2}(\Omega)\right]^{2}}+\|\mathbf{g}\|_{\left[H_{00}^{1 / 2}\left(\Gamma_{N}\right)\right]^{2}}\right\} .
$$

Proof. The linear functionals $F$ and $G$ are clearly continuous with norms bounded above by $\left(1+\kappa_{2}\right)\|\mathbf{f}\|_{\left[L^{2}(\Omega)\right]^{2}}$ and $\|\mathbf{g}\|_{\left[H_{00}^{1 / 2}\left(\Gamma_{N}\right)\right]^{2}}$, respectively. Therefore, the present proof follows from Theorem 3.3 and the well known Babuška-Brezzi theory.

\section{The Augmented miXed Finite ElEment Methods}

In this section we consider the Galerkin schemes associated to (3.4) and (3.17), define explicit finite element subspaces yielding the unique solvability of them, derive the a priori error estimates, provide the corresponding convergence results, and discuss some computational aspects.

We first let $\left\{\mathcal{T}_{h}\right\}_{h>0}$ be a regular family of triangulations of the polygonal region $\bar{\Omega}$ by triangles $T$ of diameter $h_{T}$ with mesh size $h:=\max \left\{h_{T}: \quad T \in \mathcal{T}_{h}\right\}$, and such that there holds $\bar{\Omega}:=\cup\left\{T: T \in \mathcal{T}_{h}\right\}$. Also, we assume that each point in $\bar{\Gamma}_{D} \cap \bar{\Gamma}_{N}$ becomes a vertex of $\mathcal{T}_{h}$ for all $h>0$. In addition, given an integer $\ell \geq 0$ and a subset $S$ of $\mathbb{R}^{2}$, we denote by $\mathbb{P}_{\ell}(S)$ the space of polynomials in two variables defined in $S$ of total degree at most $\ell$, and for each $T \in \mathcal{T}_{h}$ we define the local Raviart-Thomas space of order zero

$$
\mathbb{R T}_{0}(T):=\operatorname{span}\left\{\left(\begin{array}{l}
1 \\
0
\end{array}\right),\left(\begin{array}{l}
0 \\
1
\end{array}\right),\left(\begin{array}{l}
x_{1} \\
x_{2}
\end{array}\right)\right\} \subseteq\left[\mathbb{P}_{1}(T)\right]^{2},
$$

where $\left(\begin{array}{l}x_{1} \\ x_{2}\end{array}\right)$ is a generic vector of $\mathbb{R}^{2}$.

\subsection{Dirichlet boundary conditions}

Given a finite element subspace $\mathbf{H}_{0, h} \subseteq \mathbf{H}_{0}:=H_{0} \times\left[H_{0}^{1}(\Omega)\right]^{2} \times\left[L^{2}(\Omega)\right]_{\text {skew }}^{2 \times 2}$, the Galerkin scheme associated to (3.4) reads: find $\left(\boldsymbol{\sigma}_{h}, \mathbf{u}_{h}, \boldsymbol{\gamma}_{h}\right) \in \mathbf{H}_{0, h}$ such that

$$
A\left(\left(\boldsymbol{\sigma}_{h}, \mathbf{u}_{h}, \boldsymbol{\gamma}_{h}\right),\left(\boldsymbol{\tau}_{h}, \mathbf{v}_{h}, \boldsymbol{\eta}_{h}\right)\right)=F\left(\boldsymbol{\tau}_{h}, \mathbf{v}_{h}, \boldsymbol{\eta}_{h}\right) \quad \forall\left(\boldsymbol{\tau}_{h}, \mathbf{v}_{h}, \boldsymbol{\eta}_{h}\right) \in \mathbf{H}_{0, h}
$$

where $\kappa_{1}, \kappa_{2}$, and $\kappa_{3}$, being the same parameters employed in the formulation (3.4), satisfy the assumptions of Theorem 3.1. Hence, it is important to emphasize that they are independent of any meshsize associated to the finite element subspace $\mathbf{H}_{0, h}$.

Since $A$ is bounded and strongly coercive on the whole space $\mathbf{H}_{0}$ ( $c f$. Thm. 3.1), the well-posedness of (4.1) is guaranteed with any arbitrary choice of the subspace $\mathbf{H}_{0, h}$. In particular, defining, respectively, the Raviart-Thomas space of lowest order and the piecewise linear functions,

$$
H_{h}^{\boldsymbol{\sigma}}:=\left\{\boldsymbol{\tau}_{h} \in H(\operatorname{div} ; \Omega):\left.\quad \boldsymbol{\tau}_{h}\right|_{T} \in\left[\mathbb{R}_{0}(T)^{\mathrm{t}}\right]^{2} \quad \forall T \in \mathcal{T}_{h}\right\}
$$


and

$$
H_{h}^{\mathbf{u}}:=\left\{\mathbf{v}_{h} \in[C(\bar{\Omega})]^{2}:\left.\quad \mathbf{v}_{h}\right|_{T} \in\left[\mathbb{P}_{1}(T)\right]^{2} \quad \forall T \in \mathcal{T}_{h}\right\}
$$

we may take $\mathbf{H}_{0, h}:=H_{0, h}^{\boldsymbol{\sigma}} \times H_{0, h}^{\mathbf{u}} \times H_{h}^{\boldsymbol{\gamma}}$, where

$$
\begin{gathered}
H_{0, h}^{\boldsymbol{\sigma}}:=\left\{\boldsymbol{\tau}_{h} \in H_{h}^{\boldsymbol{\sigma}}: \quad \int_{\Omega} \operatorname{tr}\left(\boldsymbol{\tau}_{h}\right)=0\right\}, \\
H_{0, h}^{\mathbf{u}}:=\left\{\mathbf{v}_{h} \in H_{h}^{\mathbf{u}}: \quad \mathbf{v}_{h}=0 \quad \text { on } \quad \partial \Omega\right\},
\end{gathered}
$$

and

$$
H_{h}^{\gamma}:=\left\{\boldsymbol{\eta}_{h} \in\left[L^{2}(\Omega)\right]_{\text {skew }}^{2 \times 2}:\left.\quad \boldsymbol{\eta}_{h}\right|_{T} \in\left[\mathbb{P}_{0}(T)\right]^{2 \times 2} \quad \forall T \in \mathcal{T}_{h}\right\}
$$

In general, we have the following main result.

Theorem 4.1. Assume that the parameters $\kappa_{1}, \kappa_{2}$, and $\kappa_{3}$ satisfy the assumptions of Theorem 3.1 and let $\mathbf{H}_{0, h}$ be any finite element subspace of $\mathbf{H}_{0}$. Then, the Galerkin scheme (4.1) has a unique solution $\left(\boldsymbol{\sigma}_{h}, \mathbf{u}_{h}, \gamma_{h}\right) \in$ $\mathbf{H}_{0, h}$, and there exist positive constants $C, \tilde{C}$, independent of $\lambda$ and $h$, such that

$$
\left\|\left(\boldsymbol{\sigma}_{h}, \mathbf{u}_{h}, \boldsymbol{\gamma}_{h}\right)\right\|_{\mathbf{H}_{0}} \leq C \sup _{\substack{\left(\boldsymbol{\tau}_{h}, \mathbf{v}_{h}, \boldsymbol{\eta}_{h}\right) \in \mathbf{H}_{0, h} \\\left(\boldsymbol{\tau}_{h}, \mathbf{v}_{h}, \boldsymbol{\eta}_{h}\right) \neq 0}} \frac{\left|F\left(\boldsymbol{\tau}_{h}, \mathbf{v}_{h}, \boldsymbol{\eta}_{h}\right)\right|}{\left\|\left(\boldsymbol{\tau}_{h}, \mathbf{v}_{h}, \boldsymbol{\eta}_{h}\right)\right\|_{\mathbf{H}_{0}}} \leq C\|\mathbf{f}\|_{\left[L^{2}(\Omega)\right]^{2}},
$$

and

$$
\left\|(\boldsymbol{\sigma}, \mathbf{u}, \boldsymbol{\gamma})-\left(\boldsymbol{\sigma}_{h}, \mathbf{u}_{h}, \boldsymbol{\gamma}_{h}\right)\right\|_{\mathbf{H}_{0}} \leq \tilde{C} \inf _{\left(\boldsymbol{\tau}_{h}, \mathbf{v}_{h}, \boldsymbol{\eta}_{h}\right) \in \mathbf{H}_{0, h}}\left\|(\boldsymbol{\sigma}, \mathbf{u}, \boldsymbol{\gamma})-\left(\boldsymbol{\tau}_{h}, \mathbf{v}_{h}, \boldsymbol{\eta}_{h}\right)\right\|_{\mathbf{H}_{0}}
$$

Proof. It follows straightforward from Theorem 3.1, Lax-Milgram lemma, and Cea's estimate.

As usual, the estimate (4.7) and the approximation properties of the subspaces involved are the key ingredients to derive the corresponding rate of convergence of the Galerkin scheme. In the case of the space $\mathbf{H}_{0, h}:=$ $H_{0, h}^{\sigma} \times H_{0, h}^{\mathbf{u}} \times H_{h}^{\gamma}$ we first recall from $[10,16]$ that:

$\left(\mathrm{AP}_{h}^{\boldsymbol{\sigma}}\right)$ For each $\boldsymbol{\tau} \in\left[H^{1}(\Omega)\right]^{2 \times 2}$ with $\boldsymbol{d i v}(\boldsymbol{\tau}) \in\left[H^{1}(\Omega)\right]^{2}$ there exists $\boldsymbol{\tau}_{h} \in H_{h}^{\boldsymbol{\sigma}}$ such that

$$
\left\|\boldsymbol{\tau}-\boldsymbol{\tau}_{h}\right\|_{H(\operatorname{div} ; \Omega)} \leq C h\left\{\|\boldsymbol{\tau}\|_{\left[H^{1}(\Omega)\right]^{2 \times 2}}+\|\operatorname{div}(\boldsymbol{\tau})\|_{\left[H^{1}(\Omega)\right]^{2}}\right\} .
$$

$\left(\mathrm{AP}_{0, h}^{\mathbf{u}}\right)$ For each $\mathbf{v} \in\left[H^{2}(\Omega)\right]^{2} \cap\left[H_{0}^{1}(\Omega)\right]^{2}$ there exists $\mathbf{v}_{h} \in H_{0, h}^{\mathbf{u}}$ such that

$$
\left\|\mathbf{v}-\mathbf{v}_{h}\right\|_{\left[H^{1}(\Omega)\right]^{2}} \leq C h\|\mathbf{v}\|_{\left[H^{2}(\Omega)\right]^{2}} .
$$

$\left(\mathrm{AP}_{h}^{\gamma}\right)$ For each $\boldsymbol{\eta} \in\left[H^{1}(\Omega)\right]_{\text {skew }}^{2 \times 2}$ there exists $\boldsymbol{\eta}_{h} \in H_{h}^{\gamma}$ such that

$$
\left\|\boldsymbol{\eta}-\boldsymbol{\eta}_{h}\right\|_{\left[L^{2}(\Omega)\right]^{2 \times 2}} \leq C h\|\boldsymbol{\eta}\|_{\left[H^{1}(\Omega)\right]^{2 \times 2}} .
$$

Now, given $\boldsymbol{\tau} \in\left[H^{1}(\Omega)\right]^{2 \times 2} \cap H_{0}$ with $\operatorname{div}(\boldsymbol{\tau}) \in\left[H^{1}(\Omega)\right]^{2}$, the element $\boldsymbol{\tau}_{h} \in H_{h}^{\boldsymbol{\sigma}}$ provided by $\left(\mathrm{AP}_{h}^{\boldsymbol{\sigma}}\right)$ can be certainly decomposed as $\boldsymbol{\tau}_{h}=\boldsymbol{\tau}_{0 h}+d_{h} \mathbf{I}$, with $\boldsymbol{\tau}_{0 h} \in H_{0, h}^{\boldsymbol{\sigma}}$ and $d_{h} \in \mathbb{R}$, and hence

$$
\left\|\boldsymbol{\tau}-\boldsymbol{\tau}_{0 h}\right\|_{H(\operatorname{div} ; \Omega)}^{2} \leq\left\|\boldsymbol{\tau}-\boldsymbol{\tau}_{0 h}\right\|_{H(\operatorname{div} ; \Omega)}^{2}+2 d_{h}^{2}|\Omega|=\left\|\boldsymbol{\tau}-\boldsymbol{\tau}_{h}\right\|_{H(\operatorname{div} ; \Omega)}^{2} .
$$

It follows that, in addition to $\left(\mathrm{AP}_{h}^{\boldsymbol{\sigma}}\right)$, we also have:

$\left(\mathrm{AP}_{0, h}^{\boldsymbol{\sigma}}\right)$ For each $\boldsymbol{\tau} \in\left[H^{1}(\Omega)\right]^{2 \times 2} \cap H_{0}$ with $\operatorname{div}(\boldsymbol{\tau}) \in\left[H^{1}(\Omega)\right]^{2}$ there exists $\boldsymbol{\tau}_{h} \in H_{0, h}^{\boldsymbol{\sigma}}$ such that

$$
\left\|\boldsymbol{\tau}-\boldsymbol{\tau}_{h}\right\|_{H(\operatorname{div} ; \Omega)} \leq C h\left\{\|\boldsymbol{\tau}\|_{\left[H^{1}(\Omega)\right]^{2 \times 2}}+\|\operatorname{div}(\boldsymbol{\tau})\|_{\left[H^{1}(\Omega)\right]^{2}}\right\} .
$$


In this way, we are able to establish the following result.

Theorem 4.2. Let $(\boldsymbol{\sigma}, \mathbf{u}, \boldsymbol{\gamma}) \in \mathbf{H}_{0}$ and $\left(\boldsymbol{\sigma}_{h}, \mathbf{u}_{h}, \gamma_{h}\right) \in \mathbf{H}_{0, h}:=H_{0, h}^{\boldsymbol{\sigma}} \times H_{0, h}^{\mathbf{u}} \times H_{h}^{\boldsymbol{\gamma}}$ be the unique solutions of the continuous and discrete augmented mixed formulations (3.4) and (4.1), respectively. Assume that $\boldsymbol{\sigma} \in$ $\left[H^{r}(\Omega)\right]^{2 \times 2}, \operatorname{div}(\boldsymbol{\sigma}) \in\left[H^{r}(\Omega)\right]^{2}, \mathbf{u} \in\left[H^{r+1}(\Omega)\right]^{2}$, and $\boldsymbol{\gamma} \in\left[H^{r}(\Omega)\right]^{2 \times 2}$, for some $r \in(0,1]$. Then there exists $C>0$, independent of $\lambda$ and $h$, such that

$$
\left\|(\boldsymbol{\sigma}, \mathbf{u}, \boldsymbol{\gamma})-\left(\boldsymbol{\sigma}_{h}, \mathbf{u}_{h}, \boldsymbol{\gamma}_{h}\right)\right\|_{\mathbf{H}_{0}} \leq C h^{r}\left\{\|\boldsymbol{\sigma}\|_{\left[H^{r}(\Omega)\right]^{2 \times 2}}+\|\operatorname{div}(\boldsymbol{\sigma})\|_{\left[H^{r}(\Omega)\right]^{2}}+\|\mathbf{u}\|_{\left[H^{r+1}(\Omega)\right]^{2}}+\|\boldsymbol{\gamma}\|_{\left[H^{r}(\Omega)\right]^{2 \times 2}}\right\} .
$$

Proof. It is a consequence of Cea's estimate, approximation properties $\left(\mathrm{AP}_{0, h}^{\boldsymbol{\sigma}}\right),\left(\mathrm{AP}_{0, h}^{\mathbf{u}}\right)$, and $\left(\mathrm{AP}_{h}^{\gamma}\right)$, and suitable interpolation theorems in the corresponding function spaces.

On the other hand, it is important to remark that the null mean value condition required by the traces of the elements in $H_{0, h}^{\sigma}$ is not very convenient for the numerical implementation of (4.1). The usual way to obtain a basis of $H_{0, h}^{\sigma}$ is to start with one from the given subspace $H_{h}^{\sigma}$, and then take the $H_{0, h}^{\sigma}$-components of the latter according to the decomposition $H_{h}^{\boldsymbol{\sigma}}=H_{0, h}^{\boldsymbol{\sigma}} \oplus \mathbb{R} \mathbf{I}$, which is valid for any subspace $H_{h}^{\boldsymbol{\sigma}}$ of $H$ containing the multiples of the identity matrix. However, it is easy to see that this canonical procedure yields basis functions of $H_{0, h}^{\sigma}$ with support $\Omega$, and hence the corresponding block in the global stiffness matrix will likely become full.

In order to overcome the above difficulty, we now let $H_{h}^{\boldsymbol{\sigma}} \times H_{0, h}^{\mathbf{u}} \times H_{h}^{\gamma}$ be any finite element subspace of $H \times\left[H_{0}^{1}(\Omega)\right]^{2} \times\left[L^{2}(\Omega)\right]_{\text {skew }}^{2 \times 2}$, and suggest to consider, instead of (4.1), the modified discrete scheme: find $\left(\boldsymbol{\sigma}_{h}, \mathbf{u}_{h}, \gamma_{h}, \varphi_{h}\right) \in H_{h}^{\boldsymbol{\sigma}} \times H_{0, h}^{\mathbf{u}} \times H_{h}^{\gamma} \times \mathbb{R}$ such that

$$
\begin{aligned}
A\left(\left(\boldsymbol{\sigma}_{h}, \mathbf{u}_{h}, \boldsymbol{\gamma}_{h}\right),\left(\boldsymbol{\tau}_{h}, \mathbf{v}_{h}, \boldsymbol{\eta}_{h}\right)\right)+\varphi_{h} \int_{\Omega} \operatorname{tr}\left(\boldsymbol{\tau}_{h}\right) & =F\left(\boldsymbol{\tau}_{h}, \mathbf{v}_{h}, \boldsymbol{\eta}_{h}\right), \\
\psi_{h} \int_{\Omega} \operatorname{tr}\left(\boldsymbol{\sigma}_{h}\right) & =0
\end{aligned}
$$

for all $\left(\boldsymbol{\tau}_{h}, \mathbf{v}_{h}, \boldsymbol{\eta}_{h}, \psi_{h}\right) \in H_{h}^{\boldsymbol{\sigma}} \times H_{0, h}^{\mathbf{u}} \times H_{h}^{\boldsymbol{\gamma}} \times \mathbb{R}$. In this way, the Lagrange multiplier $\varphi_{h} \in \mathbb{R}$ and the corresponding test constants $\psi_{h} \in \mathbb{R}$ take care of the above mentioned mean value condition, whence (4.1) and (4.8) become equivalent, as it is established in the following theorem.

\section{Theorem 4.3.}

(a) Let $\left(\boldsymbol{\sigma}_{h}, \mathbf{u}_{h}, \boldsymbol{\gamma}_{h}\right) \in \mathbf{H}_{0, h}$ be the solution of (4.1). Then $\left(\boldsymbol{\sigma}_{h}, \mathbf{u}_{h}, \boldsymbol{\gamma}_{h}, 0\right)$ is a solution of (4.8).

(b) Let $\left(\boldsymbol{\sigma}_{h}, \mathbf{u}_{h}, \boldsymbol{\gamma}_{h}, \varphi_{h}\right) \in H_{h}^{\boldsymbol{\sigma}} \times H_{0, h}^{\mathbf{u}} \times H_{h}^{\boldsymbol{\gamma}} \times \mathbb{R}$ be a solution of (4.8). Then $\varphi_{h}=0$ and $\left(\boldsymbol{\sigma}_{h}, \mathbf{u}_{h}, \boldsymbol{\gamma}_{h}\right)$ is the solution of (4.1).

Proof. We first observe, according to the definition of $A$ (cf. (3.5)), that for each $(\boldsymbol{\tau}, \mathbf{v}, \boldsymbol{\eta}) \in H \times\left[H^{1}(\Omega)\right]^{2} \times$ $\left[L^{2}(\Omega)\right]_{\text {skew }}^{2 \times 2}$ there holds

$$
A((\boldsymbol{\tau}, \mathbf{v}, \boldsymbol{\eta}),(\mathbf{I}, 0,0))=\frac{1}{2(\lambda+\mu)}\left(1-\frac{\kappa_{1}}{2(\lambda+\mu)}\right) \int_{\Omega} \operatorname{tr}(\boldsymbol{\tau})+\frac{\kappa_{1}}{2(\lambda+\mu)} \int_{\Omega} \operatorname{div}(\mathbf{v}),
$$

which gives

$$
A((\boldsymbol{\tau}, \mathbf{v}, \boldsymbol{\eta}),(\mathbf{I}, 0,0))=0 \quad \forall(\boldsymbol{\tau}, \mathbf{v}, \boldsymbol{\eta}) \in H_{0} \times\left[H_{0}^{1}(\Omega)\right]^{2} \times\left[L^{2}(\Omega)\right]_{\text {skew }}^{2 \times 2} .
$$

Now, let $\left(\boldsymbol{\sigma}_{h}, \mathbf{u}_{h}, \gamma_{h}\right) \in \mathbf{H}_{0, h}$ be the solution of (4.1), and let $\left(\boldsymbol{\tau}_{h}, \mathbf{v}_{h}, \boldsymbol{\eta}_{h}\right) \in H_{h}^{\boldsymbol{\sigma}} \times H_{0, h}^{\mathbf{u}} \times H_{h}^{\gamma}$. We write $\boldsymbol{\tau}_{h}=\boldsymbol{\tau}_{0 h}+d_{h} \mathbf{I}$, with $\boldsymbol{\tau}_{0 h} \in H_{0, h}^{\boldsymbol{\sigma}}$ and $d_{h} \in \mathbb{R}$, and observe that $\left(\boldsymbol{\tau}_{0 h}, \mathbf{v}_{h}, \boldsymbol{\eta}_{h}\right) \in \mathbf{H}_{0, h}$, whence (3.6), (4.1), and (4.9) yield

$$
F\left(\boldsymbol{\tau}_{h}, \mathbf{v}_{h}, \boldsymbol{\eta}_{h}\right)=F\left(\boldsymbol{\tau}_{0 h}, \mathbf{v}_{h}, \boldsymbol{\eta}_{h}\right)=A\left(\left(\boldsymbol{\sigma}_{h}, \mathbf{u}_{h}, \boldsymbol{\gamma}_{h}\right),\left(\boldsymbol{\tau}_{0 h}, \mathbf{v}_{h}, \boldsymbol{\eta}_{h}\right)\right)=A\left(\left(\boldsymbol{\sigma}_{h}, \mathbf{u}_{h}, \boldsymbol{\gamma}_{h}\right),\left(\boldsymbol{\tau}_{h}, \mathbf{v}_{h}, \boldsymbol{\eta}_{h}\right)\right) .
$$


This identity and the fact that $\boldsymbol{\sigma}_{h}$ clearly satisfies the second equation of (4.8), show that $\left(\boldsymbol{\sigma}_{h}, \mathbf{u}_{h}, \boldsymbol{\gamma}_{h}, 0\right)$ is indeed a solution of (4.8).

Conversely, let $\left(\boldsymbol{\sigma}_{h}, \mathbf{u}_{h}, \gamma_{h}, \varphi_{h}\right) \in H_{h}^{\boldsymbol{\sigma}} \times H_{0, h}^{\mathbf{u}} \times H_{h}^{\boldsymbol{\gamma}} \times \mathbb{R}$ be a solution of (4.8). Then, taking $\left(\boldsymbol{\tau}_{h}, \mathbf{v}_{h}, \boldsymbol{\eta}_{h}\right)=$ $(\mathbf{I}, 0,0)$ in the first equation of (4.8) and using (3.6) and (4.9), we find that $\varphi_{h}=0$, whence $\left(\boldsymbol{\sigma}_{h}, \mathbf{u}_{h}, \boldsymbol{\gamma}_{h}\right)$ becomes the solution of (4.1).

We end this section by emphasizing, according to the above equivalence result, that the actual computation of the Galerkin solution $\left(\boldsymbol{\sigma}_{h}, \mathbf{u}_{h}, \gamma_{h}\right)$ must be performed through the numerical implementation of the scheme (4.8), instead of (4.1).

\subsection{Mixed boundary conditions}

In this case, and differently from (3.4), the coerciveness and the inf-sup condition satisfied, respectively, by the bilinear forms $A$ and $B$ (cf. Thm. 3.3) are not directly transferred to the discrete level. Consequently, the eventual unique solvability and stability of a Galerkin scheme associated to (3.17) do not follow either from the well-posedness provided by Theorem 3.4. Actually, as it is typical of the dual-mixed variational formulations of the form (3.17), these properties and results need to be proved for each particular choice of the corresponding finite element subspaces.

According to the above, and for reasons that will become clear below, we now introduce an independent partition $\left\{\tilde{e}_{1}, \tilde{e}_{2}, \ldots, \tilde{e}_{m}\right\}$ of the Neumann boundary $\Gamma_{N}$, define the mesh size $\tilde{h}:=\max \left\{\left|\tilde{e}_{j}\right|: j \in\{1, \ldots, m\}\right\}$, and consider a finite element subspace $\mathbf{Q}_{\tilde{h}}$ of $\mathbf{Q}:=\left[H_{00}^{1 / 2}\left(\Gamma_{N}\right)\right]^{2}$. Next, we let $\mathbf{H}_{h}:=H_{h}^{\boldsymbol{\sigma}} \times H_{D, h}^{\mathbf{u}} \times H_{h}^{\gamma}$ be any finite element subspace of $\mathbf{H}:=H \times\left[H_{\Gamma_{D}}^{1}(\Omega)\right]^{2} \times\left[L^{2}(\Omega)\right]_{\text {skew }}^{2 \times 2}$ and set the Galerkin scheme associated to (3.17): find $\left(\left(\boldsymbol{\sigma}_{h}, \mathbf{u}_{h}, \boldsymbol{\gamma}_{h}\right), \boldsymbol{\xi}_{\tilde{h}}\right) \in \mathbf{H}_{h} \times \mathbf{Q}_{\tilde{h}}$ such that

$$
\begin{aligned}
A\left(\left(\boldsymbol{\sigma}_{h}, \mathbf{u}_{h}, \boldsymbol{\gamma}_{h}\right),\left(\boldsymbol{\tau}_{h}, \mathbf{v}_{h}, \boldsymbol{\eta}_{h}\right)\right)+B\left(\left(\boldsymbol{\tau}_{h}, \mathbf{v}_{h}, \boldsymbol{\eta}_{h}\right), \boldsymbol{\xi}_{\tilde{h}}\right) & =F\left(\boldsymbol{\tau}_{h}, \mathbf{v}_{h}, \boldsymbol{\eta}_{h}\right), \\
B\left(\left(\boldsymbol{\sigma}_{h}, \mathbf{u}_{h}, \boldsymbol{\gamma}_{h}\right), \boldsymbol{\chi}_{\tilde{h}}\right) & =G\left(\boldsymbol{\chi}_{\tilde{h}}\right),
\end{aligned}
$$

for all $\left(\left(\boldsymbol{\tau}_{h}, \mathbf{v}_{h}, \boldsymbol{\eta}_{h}\right), \boldsymbol{\chi}_{\tilde{h}}\right) \in \mathbf{H}_{h} \times \mathbf{Q}_{\tilde{h}}$, where the vector of parameters $\left(\kappa_{1}, \kappa_{2}, \kappa_{3}\right)$ satisfies the assumptions indicated in Theorem 3.3 as well as the eventual ones to be needed for the well-posedness of (4.10).

In what follows, we assume that there holds the following approximation property of $\mathbf{Q}_{\tilde{h}}$ :

$\left(\operatorname{AP}_{\tilde{h}}^{\boldsymbol{\xi}}\right)$ For each $\boldsymbol{\chi} \in\left[H_{00}^{3 / 2}\left(\Gamma_{N}\right)\right]^{2}$ there exists $\boldsymbol{\chi}_{\tilde{h}} \in \mathbf{Q}_{\tilde{h}}$ such that

$$
\left\|\chi-\chi_{\tilde{h}}\right\|_{\left[H_{00}^{1 / 2}\left(\Gamma_{N}\right)\right]^{2}} \leq C \tilde{h}\|\chi\|_{\left[H_{00}^{3 / 2}\left(\Gamma_{N}\right)\right]^{2}} .
$$

Then, we use $\left(\mathrm{AP}_{\tilde{h}}^{\boldsymbol{\xi}}\right)$ to prove the discrete analogue of Lemma 2.2, which, similarly to the continuous case (cf. (3.21)), will serve to show the coerciveness of $A$ on the discrete null space of $B$.

Lemma 4.4. Let $V_{h, \tilde{h}}:=\left\{\boldsymbol{\tau}_{h} \in H_{h}^{\boldsymbol{\sigma}}:\left\langle\boldsymbol{\tau}_{h} \boldsymbol{\nu}, \boldsymbol{\chi}_{\tilde{h}}\right\rangle_{\Gamma_{N}}=0 \quad \forall \boldsymbol{\chi}_{\tilde{h}} \in \mathbf{Q}_{\tilde{h}}\right\}$. Then there exist $C_{2}, h_{0}>0$, independent of $h$ and $\tilde{h}$, such that for each $\tilde{h} \leq h_{0}$ there holds:

$$
C_{2}\left\|\boldsymbol{\tau}_{h}\right\|_{H(\operatorname{div} ; \Omega)}^{2} \leq\left\|\boldsymbol{\tau}_{0 h}\right\|_{H(\operatorname{div} ; \Omega)}^{2} \quad \forall \boldsymbol{\tau}_{h} \in V_{h, \tilde{h}}
$$

Proof. Let $\boldsymbol{\tau}_{h} \in V_{h, \tilde{h}}$ and write $\boldsymbol{\tau}_{h}=\boldsymbol{\tau}_{0 h}+d_{h} \mathbf{I}$ with $\boldsymbol{\tau}_{0 h} \in H_{0, h}^{\boldsymbol{\sigma}}$ and $d_{h} \in \mathbb{R}$. Then, given $\boldsymbol{\chi} \in\left[H_{00}^{3 / 2}\left(\Gamma_{N}\right)\right]^{2} \subseteq$ $\left[H_{00}^{1 / 2}\left(\Gamma_{N}\right)\right]^{2}$, we apply $\left(\operatorname{AP}_{\tilde{h}}^{\boldsymbol{\xi}}\right)$ and the condition defining $V_{h, \tilde{h}}$ to write

$$
\left\langle d_{h} \boldsymbol{\nu}, \boldsymbol{\chi}\right\rangle_{\Gamma_{N}}=\left\langle\boldsymbol{\tau}_{h} \boldsymbol{\nu}, \boldsymbol{\chi}\right\rangle_{\Gamma_{N}}-\left\langle\boldsymbol{\tau}_{0 h} \boldsymbol{\nu}, \boldsymbol{\chi}\right\rangle_{\Gamma_{N}}=\left\langle\boldsymbol{\tau}_{h} \boldsymbol{\nu}, \boldsymbol{\chi}-\boldsymbol{\chi}_{\tilde{h}}\right\rangle_{\Gamma_{N}}-\left\langle\boldsymbol{\tau}_{0 h} \boldsymbol{\nu}, \boldsymbol{\chi}\right\rangle_{\Gamma_{N}}
$$


which yields the existence of $\tilde{C}>0$, depending on $C\left(c f .\left(\operatorname{AP}_{\tilde{h}}^{\boldsymbol{\xi}}\right)\right), \Gamma_{N}$, the trace estimate of the normal components in $H(\operatorname{div} ; \Omega)$, and the continuous imbedding of $\left[H_{00}^{3 / 2}\left(\Gamma_{N}\right)\right]^{2}$ into $\left[H_{00}^{1 / 2}\left(\Gamma_{N}\right)\right]^{2}$, such that

$$
\begin{aligned}
\left|\left\langle d_{h} \boldsymbol{\nu}, \boldsymbol{\chi}\right\rangle_{\Gamma_{N}}\right| & \leq\|\boldsymbol{\tau} h \boldsymbol{\nu}\|_{\left[H^{-1 / 2}\left(\Gamma_{N}\right)\right]^{2}}\left\|\chi-\boldsymbol{\chi}_{\tilde{h}}\right\|_{\left[H_{00}^{1 / 2}\left(\Gamma_{N}\right)\right]^{2}}+\left\|\boldsymbol{\tau}_{0 h} \boldsymbol{\nu}\right\|_{\left[H^{-1 / 2}\left(\Gamma_{N}\right)\right]^{2}}\|\boldsymbol{\chi}\|_{\left[H_{00}^{1 / 2}\left(\Gamma_{N}\right)\right]^{2}} \\
& \leq \tilde{C}\left\{\tilde{h}\left\|\boldsymbol{\tau}_{h} \boldsymbol{\nu}\right\|_{\left[H^{-1 / 2}(\Gamma)\right]^{2}}+\left\|\boldsymbol{\tau}_{0 h} \boldsymbol{\nu}\right\|_{\left[H^{-1 / 2}(\Gamma)\right]^{2}}\right\}\|\chi\|_{\left[H_{00}^{3 / 2}\left(\Gamma_{N}\right)\right]^{2}} \\
& \leq \tilde{C}\left\{\tilde{h}\left\|\boldsymbol{\tau}_{h}\right\|_{H(\operatorname{div} ; \Omega)}+\left\|\boldsymbol{\tau}_{0 h}\right\|_{H(\operatorname{div} ; \Omega)}\right\}\|\chi\|_{\left[H_{00}^{3 / 2}\left(\Gamma_{N}\right)\right]^{2}} .
\end{aligned}
$$

In this way, we have shown that

$$
\frac{\left|\left\langle d_{h} \boldsymbol{\nu}, \boldsymbol{\chi}\right\rangle_{\Gamma_{N}}\right|}{\|\boldsymbol{\chi}\|_{\left[H_{00}^{3 / 2}\left(\Gamma_{N}\right)\right]^{2}}} \leq \tilde{C}\left\{\tilde{h}\left\|\boldsymbol{\tau}_{h}\right\|_{H(\mathbf{d i v} ; \Omega)}+\left\|\boldsymbol{\tau}_{0 h}\right\|_{H(\mathbf{d i v} ; \Omega)}\right\} \quad \forall \chi \in\left[H_{00}^{3 / 2}\left(\Gamma_{N}\right)\right]^{2}, \chi \neq 0
$$

which, noting that $\left[H^{-3 / 2}\left(\Gamma_{N}\right)\right]^{2}$ is the dual of $\left[H_{00}^{3 / 2}\left(\Gamma_{N}\right)\right]^{2}$, leads to

$$
\left|d_{h}\right| \leq \frac{1}{\|\boldsymbol{\nu}\|_{\left[H^{-3 / 2}\left(\Gamma_{N}\right)\right]^{2}}} \tilde{C}\left\{\tilde{h}\left\|\boldsymbol{\tau}_{h}\right\|_{H(\mathbf{d i v} ; \Omega)}+\left\|\boldsymbol{\tau}_{0 h}\right\|_{H(\mathbf{d i v} ; \Omega)}\right\} .
$$

This inequality and the fact that $\left\|\boldsymbol{\tau}_{h}\right\|_{H(\mathbf{d i v} ; \Omega)}^{2}=\left\|\boldsymbol{\tau}_{0 h}\right\|_{H(\mathbf{d i v} ; \Omega)}^{2}+2 d_{h}^{2}|\Omega|$ imply (4.11) for each $\tilde{h} \leq h_{0}$, where $h_{0}$ is sufficiently small. We omit further details.

At this point we remark that an explicit finite element subspace $\mathbf{Q}_{\tilde{h}}$ satisfying $\left(\mathrm{AP}_{\tilde{h}}^{\boldsymbol{\xi}}\right)$ is given by the piecewise linear functions, that is:

$$
\mathbf{Q}_{\tilde{h}}:=\left\{\boldsymbol{\chi}_{\tilde{h}} \in\left[C\left(\Gamma_{N}\right)\right]^{2} \cap\left[H_{00}^{1 / 2}\left(\Gamma_{N}\right)\right]^{2}:\left.\quad \boldsymbol{\chi}_{\tilde{h}}\right|_{\tilde{e}_{j}} \in\left[\mathbb{P}_{1}\left(\tilde{e}_{j}\right)\right]^{2} \quad \forall j \in\{1, \ldots, m\}\right\} .
$$

Also, we realize that the discrete null space of the bilinear form $B$ reduces to:

$$
\mathbf{V}_{h, \tilde{h}}:=\left\{\left(\boldsymbol{\tau}_{h}, \mathbf{v}_{h}, \boldsymbol{\eta}_{h}\right) \in \mathbf{H}_{h}:\left\langle\boldsymbol{\tau}_{h} \boldsymbol{\nu}, \boldsymbol{\chi}_{\tilde{h}}\right\rangle_{\Gamma_{N}}=0 \quad \forall \boldsymbol{\chi}_{\tilde{h}} \in \mathbf{Q}_{\tilde{h}}\right\}=V_{h, \tilde{h}} \times H_{D, h}^{\mathbf{u}} \times H_{h}^{\boldsymbol{\gamma}},
$$

which is, in general, not included in $\mathbf{V}$, the continuous null space of $B$. This fact explains the non-applicability of Lemma 2.2 in the discrete case, and the consequent need of Lemma 4.4.

Indeed, we now recall from (3.20) that for $0<\kappa_{1}<2 \mu$ and for each $(\boldsymbol{\tau}, \mathbf{v}, \boldsymbol{\eta}) \in \mathbf{H}$ there holds

$$
\begin{aligned}
A((\boldsymbol{\tau}, \mathbf{v}, \boldsymbol{\eta}),(\boldsymbol{\tau}, \mathbf{v}, \boldsymbol{\eta})) \geq & \frac{1}{2 \mu}\left(1-\frac{\kappa_{1}}{2 \mu}\right)\left\|\boldsymbol{\tau}^{\mathrm{d}}\right\|_{\left[L^{2}(\Omega)\right]^{2 \times 2}}^{2}+\left(\kappa_{1}+\kappa_{3}\right)\|\mathbf{e}(\mathbf{v})\|_{\left[L^{2}(\Omega)\right]^{2 \times 2}}^{2} \\
& +\kappa_{2}\|\operatorname{div}(\boldsymbol{\tau})\|_{\left[L^{2}(\Omega)\right]^{2}}^{2}+\kappa_{3}\|\boldsymbol{\eta}\|_{\left[L^{2}(\Omega)\right]^{2 \times 2}}^{2}-\kappa_{3}|\mathbf{v}|_{\left[H^{1}(\Omega)\right]^{2}}^{2},
\end{aligned}
$$

which, according to (2.12) (cf. Lem. 2.1), (4.13), (4.11) ( $c f$. Lem. 4.4), and Korn's first inequality (3.22), leads to

$$
A\left(\left(\boldsymbol{\tau}_{h}, \mathbf{v}_{h}, \boldsymbol{\eta}_{h}\right),\left(\boldsymbol{\tau}_{h}, \mathbf{v}_{h}, \boldsymbol{\eta}_{h}\right)\right) \geq \alpha_{2} C_{2}\left\|\boldsymbol{\tau}_{h}\right\|_{H(\operatorname{div} ; \Omega)}^{2}+\left(\kappa_{1} k_{D}-\kappa_{3}\left(1-k_{D}\right)\right)\left|\mathbf{v}_{h}\right|_{\left[H^{1}(\Omega)\right]^{2}}^{2}+\kappa_{3}\left\|\boldsymbol{\eta}_{h}\right\|_{\left.\left[L^{2}(\Omega)\right]\right]^{2 \times 2}}^{2},
$$

for all $\left(\boldsymbol{\tau}_{h}, \mathbf{v}_{h}, \boldsymbol{\eta}_{h}\right) \in \mathbf{V}_{h, \tilde{h}}$ and for all $\tilde{h} \leq h_{0}$, where $\alpha_{2}$ is the constant defined in (3.12). The rest of the analysis is similar to the continuous case ( $c f$. Sect. 3.2).

We have thus proved the following result. 
Theorem 4.5. Assume that the vector of parameters $\left(\kappa_{1}, \kappa_{2}, \kappa_{3}\right)$ satisfies the assumptions of Theorem 3.3 . Then, there exists $\bar{\alpha}>0$, independent of $\lambda, h$, and $\tilde{h}$, such that

$$
A\left(\left(\boldsymbol{\tau}_{h}, \mathbf{v}_{h}, \boldsymbol{\eta}_{h}\right),\left(\boldsymbol{\tau}_{h}, \mathbf{v}_{h}, \boldsymbol{\eta}_{h}\right)\right) \geq \bar{\alpha}\left\|\left(\boldsymbol{\tau}_{h}, \mathbf{v}_{h}, \boldsymbol{\eta}_{h}\right)\right\|_{\mathbf{H}}^{2} \quad \forall\left(\boldsymbol{\tau}_{h}, \mathbf{v}_{h}, \boldsymbol{\eta}_{h}\right) \in \mathbf{V}_{h, \tilde{h}}, \quad \forall \tilde{h} \leq h_{0} .
$$

It is important to remark here that the cost of obtaining a discrete coerciveness constant $\bar{\alpha}$, independent of $\lambda$, is given by the asymptotic equivalence (for $\tilde{h}$ sufficiently small) provided by Lemma 4.4. Otherwise, if we wanted to circumvent this, then we would have to get the corresponding lower bound of $A\left(\left(\boldsymbol{\tau}_{h}, \mathbf{v}_{h}, \boldsymbol{\eta}_{h}\right),\left(\boldsymbol{\tau}_{h}, \mathbf{v}_{h}, \boldsymbol{\eta}_{h}\right)\right)$ starting from (3.8) and (3.9), and avoiding the use of (3.10) and (3.20) (see also (4.14)). Unfortunately, in this case we obtain, instead of (4.14),

$$
\begin{aligned}
& A((\boldsymbol{\tau}, \mathbf{v}, \boldsymbol{\eta}),(\boldsymbol{\tau}, \mathbf{v}, \boldsymbol{\eta})) \geq \frac{1}{2 \mu}\left(1-\frac{\kappa_{1}}{2 \mu}\right)\|\boldsymbol{\tau}\|_{\left[L^{2}(\Omega)\right]^{2 \times 2}}^{2}+\frac{\lambda}{8 \mu(\lambda+\mu)}\left(\frac{\kappa_{1}(\lambda+2 \mu)}{\mu(\lambda+\mu)}-2\right) \int_{\Omega} \operatorname{tr}^{2}(\boldsymbol{\tau}) \\
& \quad+\left(\kappa_{1}+\kappa_{3}\right)\|\mathbf{e}(\mathbf{v})\|_{\left[L^{2}(\Omega)\right]^{2 \times 2}}^{2}+\kappa_{2}\|\operatorname{div}(\boldsymbol{\tau})\|_{\left[L^{2}(\Omega)\right]^{2}}^{2}+\kappa_{3}\|\boldsymbol{\eta}\|_{\left[L^{2}(\Omega)\right]^{2 \times 2}}^{2}-\kappa_{3}|\mathbf{v}|_{\left[H^{1}(\Omega)\right]^{2}}^{2},
\end{aligned}
$$

from which we deduce the required bound only when $\frac{2 \mu(\lambda+\mu)}{(\lambda+2 \mu)} \leq \kappa_{1}<2 \mu$. Then, it is not difficult to see that this estimate yields a coerciveness constant $\leq O\left(\frac{1}{\lambda}\right)$, which leads to the numerical locking of the scheme (4.10).

Our next goal is to show the discrete inf-sup condition of $B$ by following the analysis provided in [5]. Actually, the technical results given below ( $c f$. Lems. 4.6 and 4.7) are simple extensions of the corresponding ones in Section 3 of [5]. To this end, we now consider the specific finite element subspaces $H_{h}^{\boldsymbol{\sigma}}$ and $\mathbf{Q}_{\tilde{h}}$ defined, respectively, by (4.2) and (4.12). In addition, we let $\left\{e_{1}, e_{2}, \ldots, e_{n}\right\}$ be the partition on $\Gamma_{N}$ inherited from the triangulation $\mathcal{T}_{h}$, and define the subspace $H_{h}^{-1 / 2}$ of $\left[H^{-1 / 2}\left(\Gamma_{N}\right)\right]^{2}$ given by the piecewise constant functions, that is

which satisfies the following approximation property:

$$
H_{h}^{-1 / 2}:=\left\{\boldsymbol{\rho}_{h} \in\left[L^{2}\left(\Gamma_{N}\right)\right]^{2}:\left.\quad \boldsymbol{\rho}_{h}\right|_{e_{j}} \in\left[\mathbb{P}_{0}\left(e_{j}\right)\right]^{2} \quad \forall j \in\{1, \ldots, n\}\right\},
$$

$\left(\mathrm{AP}_{h}^{-1 / 2}\right)$ For all $s \in\left(-\frac{1}{2}, \frac{1}{2}\right]$ and for all $\boldsymbol{\rho} \in\left[H^{s}\left(\Gamma_{N}\right)\right]^{2}$ there exists $\boldsymbol{\rho}_{h} \in H_{h}^{-1 / 2}$ such that

$$
\left\|\boldsymbol{\rho}-\boldsymbol{\rho}_{h}\right\|_{\left[H^{-1 / 2}\left(\Gamma_{N}\right)\right]^{2}} \leq C h^{s+1 / 2}\|\boldsymbol{\rho}\|_{\left[H^{s}\left(\Gamma_{N}\right)\right]^{2}} .
$$

Next, the family of triangulations $\left\{\mathcal{T}_{h}\right\}_{h>0}$ is assumed to be uniformly regular near $\Gamma_{N}$, which means that there exists $C>0$, independent of $h$, such that $\left|e_{j}\right| \geq C h$ for all $j \in\{1, \ldots, n\}$, for all $h>0$. This condition implies the inverse inequality for $H_{h}^{-1 / 2}$, that is for any real numbers $s$ and $t$ with $-1 / 2 \leq s \leq t \leq 0$, there exists $C>0$ such that

$$
\left\|\boldsymbol{\rho}_{h}\right\|_{\left[H^{t}\left(\Gamma_{N}\right)\right]^{2}} \leq C h^{s-t}\left\|\boldsymbol{\rho}_{h}\right\|_{\left[H^{s}\left(\Gamma_{N}\right)\right]^{2}} \quad \forall \boldsymbol{\rho}_{h} \in H_{h}^{-1 / 2} .
$$

Lemma 4.6. There exists $\bar{\beta}_{1}>0$, independent of $h$ and $\tilde{h}$, such that for all $\boldsymbol{\chi}_{\tilde{h}} \in \mathbf{Q}_{\tilde{h}}$ there holds

$$
\sup _{\substack{\boldsymbol{\tau}_{h} \in H \\ \boldsymbol{\tau}_{h} \neq 0}} \frac{\left\langle\boldsymbol{\tau}_{h} \boldsymbol{\nu}, \boldsymbol{\chi}_{\tilde{h}}\right\rangle_{\Gamma_{N}}}{\left\|\boldsymbol{\tau}_{h}\right\|_{H(\operatorname{div} ; \Omega)}} \geq \bar{\beta}_{1} \sup _{\substack{\boldsymbol{\rho}_{h} \in H_{h}^{-1 / 2} \\ \boldsymbol{\rho}_{h} \neq 0}} \frac{\left\langle\boldsymbol{\rho}_{h}, \boldsymbol{\chi}_{\tilde{h}}\right\rangle_{\Gamma_{N}}}{\left\|\boldsymbol{\rho}_{h}\right\|_{\left[H^{-1 / 2}\left(\Gamma_{N}\right)\right]^{2}}} .
$$

Proof. It is a componentwise application of Lemma 3.2 in [5], whose proof employs a suitable auxiliary problem, and applies, mainly, elliptic regularity results, the properties of the equilibrium interpolation operator mapping $\left[H^{1}(\Omega)\right]^{2 \times 2}$ onto $H_{h}^{\boldsymbol{\sigma}}$ (see [10]), and the inverse inequality (4.16). We omit further details and refer to [5].

On the other hand, we also assume that the independent partition $\left\{\tilde{e}_{1}, \ldots, \tilde{e}_{m}\right\}$ of $\Gamma_{N}$ is uniformly regular, that is there exists $C>0$, independent of $\tilde{h}$, such that $\left|\tilde{e}_{j}\right| \geq C \tilde{h}$ for all $j \in\{1, \ldots, m\}$, for all $\tilde{h}>0$. This condition 
and the fact that $\mathbf{Q}_{\tilde{h}} \subseteq\left[H_{00}^{1}\left(\Gamma_{N}\right)\right]^{2}$ guarantees the inverse inequality for $\mathbf{Q}_{\tilde{h}}$, which means that for any real numbers $s$ and $t$ with $0 \leq s \leq t \leq 1$, there exists $C>0$ such that

$$
\left\|\chi_{\tilde{h}}\right\|_{\left[H_{00}^{t}\left(\Gamma_{N}\right)\right]^{2}} \leq C \tilde{h}^{s-t}\left\|\boldsymbol{\chi}_{\tilde{h}}\right\|_{\left[H_{00}^{s}\left(\Gamma_{N}\right)\right]^{2}} \quad \forall \boldsymbol{\chi}_{\tilde{h}} \in \mathbf{Q}_{\tilde{h}}
$$

Lemma 4.7. There exist $\bar{\beta}_{2}, C_{0}>0$, independent of $h$ and $\tilde{h}$, such that for all $h \leq C_{0} \tilde{h}$ and for all $\boldsymbol{\chi}_{\tilde{h}} \in \mathbf{Q}_{\tilde{h}}$ there holds

$$
\sup _{\substack{\boldsymbol{\rho}_{h} \in H_{h}^{-1 / 2} \\ \boldsymbol{\rho}_{h} \neq 0}} \frac{\left\langle\boldsymbol{\rho}_{h}, \boldsymbol{\chi}_{\tilde{h}}\right\rangle_{\Gamma_{N}}}{\left\|\boldsymbol{\rho}_{h}\right\|_{\left[H^{-1 / 2}\left(\Gamma_{N}\right)\right]^{2}}} \geq \bar{\beta}_{2}\left\|\chi_{\tilde{h}}\right\|_{\left[H_{00}^{1 / 2}\left(\Gamma_{N}\right)\right]^{2}} .
$$

Proof. It is a componentwise application of Lemma 3.3 in [5], whose proof also employs an auxiliary problem, and applies, mainly, elliptic regularity results, the approximation property $\left(\mathrm{AP}_{h}^{-1 / 2}\right)$, and the inverse inequality (4.17). Again, we omit details and refer to [5].

As a corollary of Lemmas 4.6 and 4.7 we can establish the following result.

Theorem 4.8. There exist $\bar{\beta}, C_{0}>0$, independent of $h$ and $\tilde{h}$, such that for all $h \leq C_{0} \tilde{h}$ and for all $\boldsymbol{\chi}_{\tilde{h}} \in \mathbf{Q}_{\tilde{h}}$ there holds

$$
\sup _{\substack{\left(\boldsymbol{\tau}_{h}, \mathbf{v}_{h}, \boldsymbol{\eta}_{h}\right) \in \mathbf{H}_{h} \\\left(\boldsymbol{\tau}_{h}, \mathbf{v}_{h}, \boldsymbol{\eta}_{h}\right) \neq 0}} \frac{B\left(\left(\boldsymbol{\tau}_{h}, \mathbf{v}_{h}, \boldsymbol{\eta}_{h}\right), \boldsymbol{\chi}_{\tilde{h}}\right)}{\left\|\left(\boldsymbol{\tau}_{h}, \mathbf{v}_{h}, \boldsymbol{\eta}_{h}\right)\right\|_{\mathbf{H}}} \geq \bar{\beta}\left\|\boldsymbol{\chi}_{\tilde{h}}\right\|_{\left[H_{00}^{1 / 2}\left(\Gamma_{N}\right)\right]^{2}} .
$$

The unique solvability and stability of (4.10) and the corresponding Cea's estimate can be established now.

Theorem 4.9. Assume that the vector of parameters $\left(\kappa_{1}, \kappa_{2}, \kappa_{3}\right)$ satisfies the assumptions of Theorem 3.3 . Let $\mathbf{H}_{h}:=H_{h}^{\boldsymbol{\sigma}} \times H_{D, h}^{\mathbf{u}} \times H_{h}^{\gamma}$ be a finite element subspace of $\mathbf{H}$ with $H_{h}^{\boldsymbol{\sigma}}$ given by (4.2), and let $\mathbf{Q}_{\tilde{h}}$ be defined by (4.12). Then, for all $\tilde{h} \leq h_{0}$ and for all $h \leq C_{0} \tilde{h}$ the Galerkin scheme (4.10) has a unique solution $\left(\left(\boldsymbol{\sigma}_{h}, \mathbf{u}_{h}, \boldsymbol{\gamma}_{h}\right), \boldsymbol{\xi}_{\tilde{h}}\right) \in \mathbf{H}_{h} \times \mathbf{Q}_{\tilde{h}}$. In addition, there exist positive constants $C, \tilde{C}$, independent of $\lambda$, $h$, and $\tilde{h}$, such that

$$
\begin{aligned}
& \left\|\left(\left(\boldsymbol{\sigma}_{h}, \mathbf{u}_{h}, \boldsymbol{\gamma}_{h}\right), \boldsymbol{\xi}_{\tilde{h}}\right)\right\|_{\mathbf{H} \times \mathbf{Q}} \\
& \leq C\left\{\sup _{\substack{\left(\boldsymbol{\tau}_{h}, \mathbf{v}_{h}, \boldsymbol{\eta}_{h}\right) \in \mathbf{H}_{h} \\
\left(\boldsymbol{\tau}_{h}, \mathbf{v}_{h}, \boldsymbol{\eta}_{h}\right) \neq 0}} \frac{\left|F\left(\boldsymbol{\tau}_{h}, \mathbf{v}_{h}, \boldsymbol{\eta}_{h}\right)\right|}{\left\|\left(\boldsymbol{\tau}_{h}, \mathbf{v}_{h}, \boldsymbol{\eta}_{h}\right)\right\|_{\mathbf{H}}}+\sup _{\substack{\boldsymbol{\chi}_{\tilde{h}} \in \mathbf{Q}_{\tilde{h}} \\
\boldsymbol{\chi}_{\tilde{h}} \neq 0}} \frac{\left|G\left(\boldsymbol{\chi}_{\tilde{h}}\right)\right|}{\left\|\chi_{\tilde{h}}\right\|_{\mathbf{Q}}}\right\} \leq C\left\{\|\mathbf{f}\|_{\left[L^{2}(\Omega)\right]^{2}}+\|\mathbf{g}\|_{\left[H^{-1 / 2}\left(\Gamma_{N}\right)\right]^{2}}\right\}
\end{aligned}
$$

and

$$
\begin{aligned}
& \left\|((\boldsymbol{\sigma}, \mathbf{u}, \boldsymbol{\gamma}), \boldsymbol{\xi})-\left(\left(\boldsymbol{\sigma}_{h}, \mathbf{u}_{h}, \boldsymbol{\gamma}_{h}\right), \boldsymbol{\xi}_{\tilde{h}}\right)\right\|_{\mathbf{H} \times \mathbf{Q}} \\
& \leq \tilde{C} \inf _{\left(\left(\boldsymbol{\tau}_{h}, \mathbf{v}_{h}, \boldsymbol{\eta}_{h}\right), \boldsymbol{\chi}_{\tilde{h}}\right) \in \mathbf{H}_{h} \times \mathbf{Q}_{\tilde{h}}}\left\|((\boldsymbol{\sigma}, \mathbf{u}, \boldsymbol{\gamma}), \boldsymbol{\xi})-\left(\left(\boldsymbol{\tau}_{h}, \mathbf{v}_{h}, \boldsymbol{\eta}_{h}\right), \boldsymbol{\chi}_{\tilde{h}}\right)\right\|_{\mathbf{H} \times \mathbf{Q}} .
\end{aligned}
$$

Proof. It is a consequence of the boundedness of $A$ and $B$ (cf. Thm. 3.3), Theorems 4.4 and 4.5, and the well known Babuška-Brezzi theory.

The corresponding rate of convergence of the Galerkin scheme (4.10) is provided next. To this end, we also assume that there holds an approximation property of $H_{D, h}^{\mathbf{u}}$, say $\left(\mathrm{AP}_{D, h}^{\mathbf{u}}\right)$, which is analogous to the one of $H_{0, h}^{\mathbf{u}}\left(\operatorname{see}\left(\mathrm{AP}_{0, h}^{\mathbf{u}}\right)\right)$. 
Theorem 4.10. Let $((\boldsymbol{\sigma}, \mathbf{u}, \boldsymbol{\gamma}), \boldsymbol{\xi}) \in \mathbf{H}$ and $\left(\left(\boldsymbol{\sigma}_{h}, \mathbf{u}_{h}, \boldsymbol{\gamma}_{h}\right), \boldsymbol{\xi}_{\tilde{h}}\right) \in \mathbf{H}_{h} \times \mathbf{Q}_{\tilde{h}}$ be the unique solutions of the continuous and discrete augmented mixed formulations (3.17) and (4.10), respectively. Assume that $\boldsymbol{\sigma} \in\left[H^{r}(\Omega)\right]^{2 \times 2}$, $\operatorname{div}(\boldsymbol{\sigma}) \in\left[H^{r}(\Omega)\right]^{2}, \mathbf{u} \in\left[H^{r+1}(\Omega)\right]^{2}, \boldsymbol{\gamma} \in\left[H^{r}(\Omega)\right]^{2 \times 2}$, and $\boldsymbol{\xi} \in\left[H_{00}^{r+1 / 2}\left(\Gamma_{N}\right)\right]^{2}$ for some $r \in(0,1]$. Then there exists $C>0$, independent of $\lambda, h$, and $\tilde{h}$, such that for all $\tilde{h} \leq h_{0}$ and for all $h \leq C_{0} \tilde{h}$ there holds

$$
\begin{aligned}
&\left\|((\boldsymbol{\sigma}, \mathbf{u}, \boldsymbol{\gamma}), \boldsymbol{\xi})-\left(\left(\boldsymbol{\sigma}_{h}, \mathbf{u}_{h}, \boldsymbol{\gamma}_{h}\right), \boldsymbol{\xi}_{\tilde{h}}\right)\right\|_{\mathbf{H} \times \mathbf{Q}} \leq C \tilde{h}^{r}\|\boldsymbol{\xi}\|_{\left[H_{00}^{r+1 / 2}\left(\Gamma_{N}\right)\right]^{2}} \\
&+C h^{r}\left\{\|\boldsymbol{\sigma}\|_{\left[H^{r}(\Omega)\right]^{2 \times 2}}+\|\operatorname{div}(\boldsymbol{\sigma})\|_{\left[H^{r}(\Omega)\right]^{2}}+\|\mathbf{u}\|_{\left[H^{r+1}(\Omega)\right]^{2}}+\|\gamma\|_{\left[H^{r}(\Omega)\right]^{2 \times 2}}\right\}
\end{aligned}
$$

Proof. It follows from Cea's estimate (4.18), approximation properties $\left(\mathrm{AP}_{h}^{\boldsymbol{\sigma}}\right),\left(\mathrm{AP}_{D, h}^{\mathbf{u}}\right),\left(\mathrm{AP}_{h}^{\boldsymbol{\gamma}}\right), \operatorname{and}\left(\mathrm{AP}_{\tilde{h}}^{\boldsymbol{\xi}}\right)$, and suitable interpolation theorems in the corresponding function spaces.

\subsection{Some computational aspects}

In order to illustrate the complexity of our augmented mixed finite element schemes, we now describe the matrix structure of the discrete system (4.8), which, according to Theorem 4.3, corresponds to the case of pure Dirichlet boundary conditions. To this end, we first let $\left\{\ell_{1}, \ell_{2}, \ldots, \ell_{\bar{N}}\right\},\left\{\mathbf{x}_{1}, \mathbf{x}_{2}, \ldots, \mathbf{x}_{\bar{n}}\right\}$, and $\left\{T_{1}, T_{2}, \ldots, T_{\bar{m}}\right\}$ be the edges, interior nodes, and triangles, respectively, of the triangulation $\mathcal{T}_{h}$. Then, we denote by $\left\{\tau_{1}, \tau_{2}, \ldots, \tau_{\bar{N}}\right\}$, $\left\{v_{1}, v_{2}, \ldots, v_{\bar{n}}\right\}$, and $\left\{\eta_{1}, \eta_{2}, \ldots, \eta_{\bar{m}}\right\}$ the canonical bases of the Raviart-Thomas space of lowest order, the piecewise linear functions vanishing on $\partial \Omega$, and the piecewise constant functions, respectively. In other words, given $j \in\{1, \ldots, \bar{N}\}, \tau_{j}:=\left(\tau_{j 1}, \tau_{j 2}\right)$ is the unique function in $H(\operatorname{div} ; \Omega):=\left\{\tau \in\left[L^{2}(\Omega)\right]^{2}: \operatorname{div}(\tau) \in L^{2}(\Omega)\right\}$ such that $\left.\tau_{j}\right|_{T} \in \mathbb{R T}_{0}(T)^{\mathrm{t}}$ for all $T \in \mathcal{T}_{h}, \tau_{j} \cdot \boldsymbol{\nu}=1$ on $\ell_{j}$, and $\tau_{j} \cdot \boldsymbol{\nu}=0$ on $\ell_{i}$ for all $i \neq j$. Also, given $j \in\{1, \ldots, \bar{n}\}$, $v_{j}$ is the unique function in $C(\bar{\Omega})$ such that $\left.v_{j}\right|_{T} \in \mathbb{P}_{1}(T)$ for all $T \in \mathcal{T}_{h}, v_{j}=0$ on $\partial \Omega, v_{j}\left(\mathbf{x}_{j}\right)=1$, and $v_{j}\left(\mathbf{x}_{i}\right)=0$ for all $i \neq j$. Similarly, given $j \in\{1, \ldots, \bar{m}\}, \eta_{j}$ is the unique function in $L^{2}(\Omega)$ such that $\eta_{j}=1$ on $T_{j}$ and $\eta_{j}=0$ on $T_{i}$ for all $i \neq j$.

It follows easily that the corresponding canonical bases of the spaces $H_{h}^{\boldsymbol{\sigma}}, H_{0, h}^{\mathbf{u}}$, and $H_{h}^{\gamma}$ are given, respectively, by the following sets:

$$
\begin{gathered}
\left\{\left(\begin{array}{cc}
\tau_{j 1} & \tau_{j 2} \\
0 & 0
\end{array}\right): \quad j \in\{1, \ldots, \bar{N}\}\right\} \cup\left\{\left(\begin{array}{cc}
0 & 0 \\
\tau_{j 1} & \tau_{j 2}
\end{array}\right): \quad j \in\{1, \ldots, \bar{N}\}\right\}, \\
\left\{\left(\begin{array}{c}
v_{j} \\
0
\end{array}\right): \quad j \in\{1, \ldots, \bar{n}\}\right\} \cup\left\{\left(\begin{array}{c}
0 \\
v_{j}
\end{array}\right): \quad j \in\{1, \ldots, \bar{n}\}\right\},
\end{gathered}
$$

and

$$
\left\{\left(\begin{array}{cc}
0 & \eta_{j} \\
-\eta_{j} & 0
\end{array}\right): \quad j \in\{1, \ldots, \bar{m}\}\right\} .
$$

At this point we compare the dimensions of the subspaces $H_{h}^{\boldsymbol{\sigma}} \times H_{0, h}^{\mathbf{u}} \times H_{h}^{\gamma}$ and PEERS (cf. [1]) when uniform triangulations are utilized. In fact, we first observe that in this case each interior edge (resp. interior node) belongs to 2 (resp. 6) triangles, which yields corresponding correction factors of $\frac{1}{2}$ and $\frac{1}{6}$ when counting the global number of degrees of freedom (DOF) in terms of the number of triangles $\bar{m}$. Then, it is not difficult to see that in the augmented scheme (4.8) the parameter DOF behaves asymptotically as $5 \bar{m}$, whereas this behaviour is given by $7.5 \bar{m}$ when PEERS is used in the Galerkin scheme of the non-augmented formulation (2.10). In other words, the discrete system using PEERS introduces at large $50 \%$ more unknowns than our approach at each mesh, and therefore the present augmented method becomes a much cheaper alternative. In addition, it is also important to note that the polynomial degrees involved in the definition of $H_{h}^{\boldsymbol{\sigma}} \times H_{0, h}^{\mathbf{u}} \times H_{h}^{\gamma}$, being 1 , 1 and 0 , yield simpler computations than for the PEERS subspace, whose polynomial degrees are 2,0 , and 1 , respectively. 
We now go back to the discrete system (4.8). According to (4.19), (4.20), and (4.21), there exist $\mathcal{S}_{0}:=$ $\left(\sigma^{1}, \ldots, \sigma^{\bar{N}}\right)^{\mathrm{t}}, \mathcal{S}^{0}:=\left(\sigma_{1}, \ldots, \sigma_{\bar{N}}\right)^{\mathrm{t}} \in \mathbb{R}^{\bar{N}}, \mathcal{U}_{0}:=\left(u^{1}, \ldots, u^{\bar{n}}\right)^{\mathrm{t}}, \mathcal{U}^{0}:=\left(u_{1}, \ldots, u_{\bar{n}}\right)^{\mathrm{t}} \in \mathbb{R}^{\bar{n}}$, and $\mathcal{G}:=\left(g_{1}, \ldots, g_{\bar{m}}\right)^{\mathrm{t}} \in$ $\mathbb{R}^{\bar{m}}$, such that the first three components of the solution of (4.8) can be written as

$$
\begin{gathered}
\boldsymbol{\sigma}_{h}:=\sum_{j=1}^{\bar{N}} \sigma^{j}\left(\begin{array}{cc}
\tau_{j 1} & \tau_{j 2} \\
0 & 0
\end{array}\right)+\sum_{j=1}^{\bar{N}} \sigma_{j}\left(\begin{array}{cc}
0 & 0 \\
\tau_{j 1} & \tau_{j 2}
\end{array}\right), \\
\mathbf{u}_{h}:=\sum_{j=1}^{\bar{n}} u^{j}\left(\begin{array}{c}
v_{j} \\
0
\end{array}\right)+\sum_{j=1}^{\bar{n}} u_{j}\left(\begin{array}{c}
0 \\
v_{j}
\end{array}\right),
\end{gathered}
$$

and

$$
\gamma_{h}:=\sum_{j=1}^{\bar{m}} g_{j}\left(\begin{array}{cc}
0 & \eta_{j} \\
-\eta_{j} & 0
\end{array}\right) .
$$

In this way, the unknown vector of the linear system arising from (4.8) is given by

$$
\left[\begin{array}{c}
\mathcal{S}_{0} \\
\mathcal{S}^{0} \\
\mathcal{U}_{0} \\
\mathcal{U}^{0} \\
\mathcal{G} \\
\varphi_{h}
\end{array}\right] \in \mathbb{R}^{\bar{M}}
$$

where $\bar{M}:=2(\bar{N}+\bar{n})+\bar{m}+1$.

Now, in order to compute the associated stiffness matrix, we first observe from the definition of the bilinear form $A$ (cf. (3.5)), and using some formulae provided in Section 2, that the following identities hold:

$$
\begin{aligned}
& A\left((\boldsymbol{\sigma}, \mathbf{0}, \mathbf{0}),(\boldsymbol{\tau}, \mathbf{0}, \mathbf{0})=\frac{1}{2 \mu}\left(1-\frac{\kappa_{1}}{2 \mu}\right) \int_{\Omega} \boldsymbol{\sigma}: \boldsymbol{\tau}+\kappa_{2} \int_{\Omega} \operatorname{div}(\boldsymbol{\sigma}) \cdot \operatorname{div}(\boldsymbol{\tau})\right. \\
& -\frac{\lambda}{4 \mu(\lambda+\mu)}\left(1+\frac{\kappa_{1}}{\mu}\left(\frac{\lambda}{2(\lambda+\mu)}-1\right)\right) \int_{\Omega} \operatorname{tr}(\boldsymbol{\sigma}) \operatorname{tr}(\boldsymbol{\tau}), \\
& A((\boldsymbol{\sigma}, \mathbf{0}, \mathbf{0}),(\mathbf{0}, \mathbf{v}, \mathbf{0}))=-\int_{\Omega} \mathbf{v} \cdot \operatorname{div}(\boldsymbol{\sigma})-\frac{\kappa_{1}}{2 \mu} \int_{\Omega} \boldsymbol{\sigma}: \mathbf{e}(\mathbf{v}) \\
& +\frac{\kappa_{1} \lambda}{4 \mu(\lambda+\mu)} \int_{\Omega} \operatorname{tr}(\boldsymbol{\sigma}) \operatorname{div}(\mathbf{v}) \\
& A((\boldsymbol{\sigma}, \mathbf{0}, \mathbf{0}),(\mathbf{0}, \mathbf{0}, \boldsymbol{\eta}))=-\int_{\Omega} \boldsymbol{\eta}: \boldsymbol{\sigma}, \\
& A((\mathbf{0}, \mathbf{u}, \mathbf{0}),(\mathbf{0}, \mathbf{v}, \mathbf{0}))=\left(\frac{\kappa_{1}-\kappa_{3}}{2}\right) \int_{\Omega} \nabla \mathbf{u}: \nabla \mathbf{v}+\left(\frac{\kappa_{1}+\kappa_{3}}{2}\right) \int_{\Omega} \nabla \mathbf{u}:(\nabla \mathbf{v})^{\mathrm{t}} \\
& A((\mathbf{0}, \mathbf{u}, \mathbf{0}),(\mathbf{0}, \mathbf{0}, \boldsymbol{\eta}))=-\kappa_{3} \int_{\Omega} \nabla \mathbf{u}: \boldsymbol{\eta},
\end{aligned}
$$

and

$$
A((\mathbf{0}, \mathbf{0}, \boldsymbol{\gamma}),(\mathbf{0}, \mathbf{0}, \boldsymbol{\eta}))=\kappa_{3} \int_{\Omega} \boldsymbol{\gamma}: \boldsymbol{\eta}
$$


Then, applying (4.25) to the elements of the canonical basis (4.19), we find that the corresponding block of the stiffness matrix is given by $\left[\begin{array}{cc}\mathbf{A} & \mathbf{C} \\ \mathbf{C}^{\mathrm{t}} & \mathbf{B}\end{array}\right]$, where

$$
\begin{aligned}
& \mathbf{A}=\frac{1}{2 \mu}\left(1-\frac{\kappa_{1}}{2 \mu}\right) \mathbf{A}^{\boldsymbol{\tau}}+\kappa_{2} \mathbf{A}^{\mathrm{div}}-\frac{\lambda}{4 \mu(\lambda+\mu)}\left(1+\frac{\kappa_{1}}{\mu}\left(\frac{\lambda}{2(\lambda+\mu)}-1\right)\right) \mathbf{A}^{1,1}, \\
& \mathbf{B}=\frac{1}{2 \mu}\left(1-\frac{\kappa_{1}}{2 \mu}\right) \mathbf{A}^{\boldsymbol{\tau}}+\kappa_{2} \mathbf{A}^{\mathrm{div}}-\frac{\lambda}{4 \mu(\lambda+\mu)}\left(1+\frac{\kappa_{1}}{\mu}\left(\frac{\lambda}{2(\lambda+\mu)}-1\right)\right) \mathbf{A}^{2,2},
\end{aligned}
$$

and

$$
\mathbf{C}=-\frac{\lambda}{4 \mu(\lambda+\mu)}\left(1+\frac{\kappa_{1}}{\mu}\left(\frac{\lambda}{2(\lambda+\mu)}-1\right)\right) \mathbf{A}^{1,2}
$$

with the matrices $\mathbf{A}^{\boldsymbol{\tau}}:=\left(\mathbf{A}_{i j}^{\boldsymbol{\tau}}\right), \mathbf{A}^{\text {div }}:=\left(\mathbf{A}_{i j}^{\text {div }}\right), \mathbf{A}^{1,1}:=\left(\mathbf{A}_{i j}^{1,1}\right), \mathbf{A}^{1,2}:=\left(\mathbf{A}_{i j}^{1,2}\right)$, and $\mathbf{A}^{2,2}:=\left(\mathbf{A}_{i j}^{2,2}\right) \in \mathbb{R}^{\bar{N} \times \bar{N}}$, being defined as

$$
\begin{gathered}
\mathbf{A}_{i j}^{\boldsymbol{\tau}}:=\int_{\Omega} \tau_{i} \cdot \tau_{j}, \quad \mathbf{A}_{i j}^{\mathrm{div}}:=\int_{\Omega} \operatorname{div}\left(\tau_{i}\right) \operatorname{div}\left(\tau_{j}\right), \\
\mathbf{A}_{i j}^{1,1}:=\int_{\Omega} \tau_{i 1} \tau_{j 1}, \quad \mathbf{A}_{i j}^{1,2}:=\int_{\Omega} \tau_{i 1} \tau_{j 2}, \quad \text { and } \quad \mathbf{A}_{i j}^{2,2}:=\int_{\Omega} \tau_{i 2} \tau_{j 2} .
\end{gathered}
$$

Note that the entries of these matrices are possibly non null only for those indexes $i$ and $j$ such that the edges $\ell_{i}$ and $\ell_{j}$ form part of a common triangle in $\mathcal{T}_{h}$. In addition, observe that $\mathbf{A}^{\boldsymbol{\tau}}, \mathbf{A}^{\text {div }}, \mathbf{A}^{1,1}$, and $\mathbf{A}^{2,2}$, and hence $\mathbf{A}$ and $\mathbf{B}$, are all symmetric.

Now, applying (4.26) to the elements of the canonical bases (4.19) and (4.20), we find that the corresponding block of the stiffness matrix is given by $\left[\begin{array}{ll}\mathbf{D} & \tilde{\mathbf{E}} \\ \mathbf{E} & \mathbf{G}\end{array}\right]$, where

$$
\begin{aligned}
\mathbf{D}:=-\mathbf{D}^{\mathrm{div}} & +\frac{\kappa_{1}}{2 \mu}\left(\frac{\lambda}{2(\lambda+\mu)}-1\right) \mathbf{D}^{1,1}-\frac{\kappa_{1}}{4 \mu} \mathbf{D}^{2,2}, \\
\mathbf{E} & :=-\frac{\kappa_{1}}{4 \mu} \mathbf{D}^{1,2}+\frac{\kappa_{1} \lambda}{4 \mu(\lambda+\mu)} \mathbf{D}^{2,1} \\
\tilde{\mathbf{E}} & :=\frac{\kappa_{1} \lambda}{4 \mu(\lambda+\mu)} \mathbf{D}^{1,2}-\frac{\kappa_{1}}{4 \mu} \mathbf{D}^{2,1}
\end{aligned}
$$

and

$$
\mathbf{G}:=-\mathbf{D}^{\mathrm{div}}-\frac{\kappa_{1}}{4 \mu} \mathbf{D}^{1,1}+\frac{\kappa_{1}}{2 \mu}\left(\frac{\lambda}{2(\lambda+\mu)}-1\right) \mathbf{D}^{2,2},
$$

with the matrices $\mathbf{D}^{\mathrm{div}}:=\left(\mathbf{D}_{i j}^{\mathrm{div}}\right), \mathbf{D}^{1,1}:=\left(\mathbf{D}_{i j}^{1,1}\right), \mathbf{D}^{1,2}:=\left(\mathbf{D}_{i j}^{1,2}\right), \mathbf{D}^{2,1}:=\left(\mathbf{D}_{i j}^{2,1}\right)$, and $\mathbf{D}^{2,2}:=\left(\mathbf{D}_{i j}^{2,2}\right) \in \mathbb{R}^{\bar{n} \times \bar{N}}$, being defined as

$$
\begin{gathered}
\mathbf{D}_{i j}^{\mathrm{div}}:=\int_{\Omega} v_{i} \operatorname{div}\left(\tau_{j}\right), \quad \mathbf{D}_{i j}^{1,1}:=\int_{\Omega} \frac{\partial v_{i}}{\partial x_{1}} \tau_{j 1}, \\
\mathbf{D}_{i j}^{1,2}:=\int_{\Omega} \frac{\partial v_{i}}{\partial x_{1}} \tau_{j 2}, \quad \mathbf{D}_{i j}^{2,1}:=\int_{\Omega} \frac{\partial v_{i}}{\partial x_{2}} \tau_{j 1}, \quad \text { and } \quad \mathbf{D}_{i j}^{2,2}:=\int_{\Omega} \frac{\partial v_{i}}{\partial x_{2}} \tau_{j 2} .
\end{gathered}
$$

Note that the entries of these matrices are possibly non null only for those indexes $i$ and $j$ such that the vertex $\mathbf{x}_{i}$ and the edge $\ell_{j}$ belong to a common triangle in $\mathcal{T}_{h}$.

Also, applying (4.28) to the elements of the canonical basis (4.20), we find that the corresponding block of the stiffness matrix is given by $\left[\begin{array}{cc}\mathbf{J} & \mathbf{L} \\ \mathbf{L}^{\mathrm{t}} & \mathbf{K}\end{array}\right]$, where

$$
\mathbf{J}:=\kappa_{1} \mathbf{J}^{1,1}+\left(\frac{\kappa_{1}-\kappa_{3}}{2}\right) \mathbf{J}^{2,2}, \quad \mathbf{K}:=\left(\frac{\kappa_{1}-\kappa_{3}}{2}\right) \mathbf{J}^{1,1}+\kappa_{1} \mathbf{J}^{2,2},
$$


and

$$
\mathbf{L}:=\left(\frac{\kappa_{1}+\kappa_{3}}{2}\right) \mathbf{J}^{2,1}
$$

with the matrices $\mathbf{J}^{1,1}:=\left(\mathbf{J}_{i j}^{1,1}\right), \mathbf{J}^{2,2}:=\left(\mathbf{J}_{i j}^{2,2}\right)$, and $\mathbf{J}^{2,1}:=\left(\mathbf{J}_{i j}^{2,1}\right) \in \mathbb{R}^{\bar{n} \times \bar{n}}$, being defined as

$$
\mathbf{J}_{i j}^{1,1}:=\int_{\Omega} \frac{\partial v_{i}}{\partial x_{1}} \frac{\partial v_{j}}{\partial x_{1}}, \quad \mathbf{J}_{i j}^{2,2}:=\int_{\Omega} \frac{\partial v_{i}}{\partial x_{2}} \frac{\partial v_{j}}{\partial x_{2}}, \quad \text { and } \quad \mathbf{J}_{i j}^{2,1}:=\int_{\Omega} \frac{\partial v_{i}}{\partial x_{2}} \frac{\partial v_{j}}{\partial x_{1}}
$$

Note that the entries of these matrices are possibly non null only for those indexes $i$ and $j$ such that the vertices $\mathbf{x}_{i}$ and $\mathbf{x}_{j}$ belong to a common triangle in $\mathcal{T}_{h}$. In addition, observe that $\mathbf{J}^{1,1}$ and $\mathbf{J}^{2,2}$, and hence $\mathbf{J}$ and $\mathbf{K}$, are all symmetric.

Next, applying (4.27) to the elements of the canonical bases (4.19) and (4.21), we find that the corresponding block of the stiffness matrix is given by $\left[\begin{array}{ll}\mathbf{P} & \mathbf{Q}\end{array}\right]$, where the matrices $\mathbf{P}:=\left(\mathbf{P}_{i j}\right)$ and $\mathbf{Q}:=\left(\mathbf{Q}_{i j}\right) \in \mathbb{R}^{\bar{m} \times N}$ are defined as

$$
\mathbf{P}_{i j}:=-\int_{\Omega} \eta_{i} \tau_{j 2}=-\int_{T_{i}} \tau_{j 2} \quad \text { and } \quad \mathbf{Q}_{i j}:=\int_{\Omega} \eta_{i} \tau_{j 1}=\int_{T_{i}} \tau_{j 1} .
$$

Note that the entries of $\mathbf{P}$ and $\mathbf{Q}$ are possibly non null only for the indexes $i$ and $j$ such that the edge $\ell_{j}$ form part of triangle $T_{i}$.

Similarly, applying (4.29) to the elements of the canonical bases (4.20) and (4.21), we find that the corresponding block of the stiffness matrix is given by $\left[\begin{array}{ll}\mathbf{S} & \mathbf{T}\end{array}\right]$, where the matrices $\mathbf{S}:=\left(\mathbf{S}_{i j}\right)$ and $\mathbf{T}:=\left(\mathbf{T}_{i j}\right)$ $\in \mathbb{R}^{\bar{m} \times \bar{n}}$ are defined as

$$
\mathbf{S}_{i j}:=-\kappa_{3} \int_{\Omega} \eta_{i} \frac{\partial v_{j}}{\partial x_{2}}=-\kappa_{3} \int_{T_{i}} \frac{\partial v_{j}}{\partial x_{2}} \quad \text { and } \quad \mathbf{T}_{i j}:=\kappa_{3} \int_{\Omega} \eta_{i} \frac{\partial v_{j}}{\partial x_{1}}=\kappa_{3} \int_{T_{i}} \frac{\partial v_{j}}{\partial x_{1}} .
$$

Observe now that the entries of $\mathbf{S}$ and $\mathbf{T}$ are possibly non null only for the indexes $i$ and $j$ such that the vertex $\mathbf{x}_{j}$ belongs to triangle $T_{i}$.

On the other hand, applying (4.30) to the elements of the canonical basis (4.21), we obtain that the corresponding block of the stiffness matrix is given by

$$
\mathbf{U}:=2 \kappa_{3} \operatorname{diag}\left(\left|T_{1}\right|,\left|T_{2}\right|, \ldots,\left|T_{\bar{m}}\right|\right) \in \mathbb{R}^{\bar{m} \times \bar{m}} .
$$

The stiffness matrix is completed with the vectors $\mathbf{V}:=\left(\mathbf{V}_{i}\right)$ and $\mathbf{W}:=\left(\mathbf{W}_{i}\right) \in \mathbb{R}^{\bar{N} \times 1}$ arising from the terms involving the Lagrange multiplier $\varphi_{h}$, which are defined as

$$
\mathbf{V}_{i}:=\int_{\Omega} \tau_{i 1} \quad \text { and } \quad \mathbf{W}_{i}:=\int_{\Omega} \tau_{i 2} .
$$

Summarizing all the above, the discrete system (4.8) can be rewritten in matrix form as follows:

$$
\left[\begin{array}{cccccc}
\mathbf{A} & \mathbf{C} & -\mathbf{D}^{\mathrm{t}} & -\mathbf{E}^{\mathrm{t}} & -\mathbf{P}^{\mathrm{t}} & \mathbf{V} \\
\mathbf{C}^{\mathrm{t}} & \mathbf{B} & -\tilde{\mathbf{E}}^{\mathrm{t}} & -\mathbf{G}^{\mathbf{t}} & -\mathbf{Q}^{\mathrm{t}} & \mathbf{W} \\
\mathbf{D} & \tilde{\mathbf{E}} & \mathbf{J} & \mathbf{L} & -\mathbf{S}^{\mathrm{t}} & \mathbf{0} \\
\mathbf{E} & \mathbf{G} & \mathbf{L}^{\mathrm{t}} & \mathbf{K} & -\mathbf{T}^{\mathrm{t}} & \mathbf{0} \\
\mathbf{P} & \mathbf{Q} & \mathbf{S} & \mathbf{T} & \mathbf{U} & \mathbf{0} \\
\mathbf{V}^{\mathrm{t}} & \mathbf{W}^{\mathrm{t}} & 0 & 0 & 0 & 0
\end{array}\right]\left[\begin{array}{c}
\mathcal{S}_{0} \\
\mathcal{S}^{0} \\
\mathcal{U}_{0} \\
\mathcal{U}^{0} \\
\mathcal{G} \\
\varphi_{h}
\end{array}\right]=\left[\begin{array}{l}
\mathbf{F}^{1, d} \\
\mathbf{F}^{2, d} \\
\mathbf{F}^{1} \\
\mathbf{F}^{2} \\
\mathbf{0} \\
0
\end{array}\right]
$$


where, according to the definition of the functional $F(c f .(3.6))$ and setting $\mathbf{f}:=\left(f_{1}, f_{2}\right)^{\mathrm{t}}$, the vectors $\mathbf{F}^{1, d}:=$ $\left(\mathbf{F}_{i}^{1, d}\right), \mathbf{F}^{2, d}:=\left(\mathbf{F}_{i}^{2, d}\right) \in \mathbb{R}^{\bar{N} \times 1}$, and $\mathbf{F}^{1}:=\left(\mathbf{F}_{i}^{1}\right), \mathbf{F}^{2}:=\left(\mathbf{F}_{i}^{2}\right) \in \mathbb{R}^{\bar{n} \times 1}$, become

$$
\begin{gathered}
\mathbf{F}_{i}^{1, d}:=-\kappa_{2} \int_{\Omega} f_{1} \operatorname{div}\left(\tau_{i}\right), \quad \mathbf{F}_{i}^{2, d}:=-\kappa_{2} \int_{\Omega} f_{2} \operatorname{div}\left(\tau_{i}\right), \\
\mathbf{F}_{i}^{1}:=\int_{\Omega} f_{1} v_{i}, \quad \text { and } \quad \mathbf{F}_{i}^{2}:=\int_{\Omega} f_{2} v_{i} .
\end{gathered}
$$

Since $\mathbf{U}$ is diagonal and invertible, the vector $\mathcal{G}$ can be easily eliminated from (4.42), thus yielding a reduced system.

\section{Numerical RESUlts}

In this section we provide several numerical results illustrating the performance of the augmented mixed finite element scheme (4.8) on a finite sequence of uniform triangulations of the domain.

We begin with some notations. As indicated in Section 4.3, DOF stands for the total number of degrees of freedom (unknowns) of (4.8), which behaves asymptotically as $5 \bar{m}$, where $\bar{m}$ is the number of triangles of each triangulation. Also, the individual and total errors are denoted by

$$
e(\boldsymbol{\sigma}):=\left\|\boldsymbol{\sigma}-\boldsymbol{\sigma}_{h}\right\|_{H(\operatorname{div} ; \Omega)}, \quad e(\mathbf{u}):=\left\|\mathbf{u}-\mathbf{u}_{h}\right\|_{\left[H^{1}(\Omega)\right]^{2}}, \quad e(\boldsymbol{\gamma}):=\left\|\boldsymbol{\gamma}-\boldsymbol{\gamma}_{h}\right\|_{\left[L^{2}(\Omega)\right]^{2 \times 2}},
$$

and

$$
e(\boldsymbol{\sigma}, \mathbf{u}, \boldsymbol{\gamma}):=\left\{[e(\boldsymbol{\sigma})]^{2}+[e(\mathbf{u})]^{2}+[e(\boldsymbol{\gamma})]^{2}\right\}^{1 / 2}
$$

Next, we recall that given the Young modulus $E$ and the Poisson ratio $\nu$ of a linear elastic material, the corresponding Lamé constants are defined as

$$
\mu:=\frac{E}{2(1+\nu)} \quad \text { and } \quad \lambda:=\frac{E \nu}{(1+\nu)(1-2 \nu)}
$$

Then, in order to emphasize the convergence of our augmented scheme (4.8) independently of how large $\lambda$ becomes (locking-free property), in the examples below we fix $E=1$ and take $\nu=0.4900$ and $\nu=0.4999$, which yield the following values of $\mu$ and $\lambda$ :

\begin{tabular}{c|c|r}
$\nu$ & \multicolumn{1}{|c|}{$\mu$} & \multicolumn{1}{|c}{$\lambda$} \\
\hline 0.4900 & 0.3356 & 16.4430 \\
0.4999 & 0.3333 & 1666.4444
\end{tabular}

In addition, in order to show the robustness of the method with respect to the parameters $\kappa_{1}, \kappa_{2}$, and $\kappa_{3}$, and according to our analysis in Section 3.1, we consider the feasible choices described in (3.15) and (3.16), that is

$$
\left(\kappa_{1}, \kappa_{2}, \kappa_{3}\right)=\left(\mu, \frac{1}{2 \mu}, \frac{\mu}{2}\right) \quad \text { and } \quad\left(\kappa_{1}, \kappa_{2}, \kappa_{3}\right)=\left(\frac{3 \mu}{2}, \frac{1}{4 \mu}, \mu\right) .
$$

We now specify the four examples to be presented here. We take $\Omega$ as the square domain $] 0,1[\times] 0,1[$ and chose the datum $\mathbf{f}$ so that the Poisson ratio $\nu$ and the exact solution $\mathbf{u}$ are given as follows:

\begin{tabular}{c|c|c} 
ExAMPLE & $\nu$ & $\mathbf{u}\left(x_{1}, x_{2}\right)$ \\
\hline 1 & 0.4900 & $\left(x_{1}^{3}\left(1-x_{1}\right)^{2} x_{2}^{3}\left(1-x_{2}\right)^{2}, x_{1}^{3}\left(1-x_{1}\right)^{2} x_{2}^{3}\left(1-x_{2}\right)^{2}\right)^{\mathrm{t}}$ \\
2 & 0.4999 & $\left(x_{1}^{3}\left(1-x_{1}\right)^{2} x_{2}^{3}\left(1-x_{2}\right)^{2}, x_{1}^{3}\left(1-x_{1}\right)^{2} x_{2}^{3}\left(1-x_{2}\right)^{2}\right)^{\mathrm{t}}$ \\
3 & 0.4900 & $\left(\sin \left(\pi x_{1}\right) \sin \left(\pi x_{2}\right), \sin \left(\pi x_{1}\right) \sin \left(\pi x_{2}\right)\right)^{\mathrm{t}}$ \\
4 & 0.4999 & $\left(\sin \left(\pi x_{1}\right) \sin \left(\pi x_{2}\right), \sin \left(\pi x_{1}\right) \sin \left(\pi x_{2}\right)\right)^{\mathrm{t}}$
\end{tabular}


Actually, according to (2.1) and (2.2) we have $\boldsymbol{\sigma}=\lambda \operatorname{div}(\mathbf{u}) \mathbf{I}+2 \mu \mathbf{e}(\mathbf{u})$, and hence simple computations show that $\mathbf{f}:=-\operatorname{div}(\boldsymbol{\sigma})=-(\lambda+\mu) \nabla(\operatorname{div} \mathbf{u})-\mu \Delta \mathbf{u}$. Further, we also recall from Section 2 that the rotation $\gamma$ is defined as $\left.\frac{1}{2}\left(\nabla \mathbf{u}-(\nabla \mathbf{u})^{\mathrm{t}}\right)\right)$.

The numerical results given below were obtained in a Compaq Alpha ES40 Parallel Computer using a fortran code. The linear system (4.42) is implemented in this code exactly as explained in Section 4.3, and it is solved by a direct method. In addition, the individual errors are computed on each triangle using a Gaussian quadrature rule. Given a uniform initial triangulation of $\Omega$, each subsequent mesh is obtained from the previous one by dividing each triangle into the four ones arising after joining the middle points of its sides. The number of unknowns DOF and the number of triangles $\bar{m}$ of the uniform meshes employed in the computations are displayed in the following array, where one can also verify, as already mentioned, that the ratio DOF $/ \bar{m}$ approaches 5 as $\bar{m}$ (and hence DOF) increases:

\begin{tabular}{c|c|c|c} 
MESH & DOF & $\bar{m}$ & DOF $/ \bar{m}$ \\
\hline 1 & 643 & 128 & 5.0234 \\
2 & 1003 & 200 & 5.0150 \\
3 & 1443 & 288 & 5.0104 \\
4 & 1963 & 392 & 5.0077 \\
5 & 2563 & 512 & 5.0059 \\
6 & 3243 & 648 & 5.0046 \\
7 & 4003 & 800 & 5.0038 \\
8 & 4843 & 968 & 5.0031 \\
9 & 5763 & 1152 & 5.0026 \\
10 & 6763 & 1352 & 5.0022 \\
11 & 7843 & 1568 & 5.0019 \\
12 & 9003 & 1800 & 5.0017 \\
13 & 10243 & 2048 & 5.0015 \\
14 & 11563 & 2312 & 5.0013 \\
15 & 12963 & 2592 & 5.0012
\end{tabular}

In Tables 1 up to 4 we present the individual and total errors of each example for this sequence of uniform meshes. We first remark that there are no significant differences between the results obtained with the two choices of parameters $\left(\kappa_{1}, \kappa_{2}, \kappa_{3}\right)$, which numerically shows the corresponding robustness of our augmented mixed finite element scheme. Hence, we consider just one of these sets of parameters in each case to display in Figures 1 up to 4 the log-log curves of the number of unknowns DOF versus the meshsize $h$ and the errors. We observe there that the rate of convergence $O(h)$ predicted by Theorem 4.2 (when $r=1$ ) is attained in all the examples, independently of the value of $\nu$ (and hence of $\lambda$ ), which confirms not only the a priori error estimate provided by that theorem but also the locking-free character of our method. We also notice in Examples 1 and 2 that the convergence of $e(\mathbf{u})$ is even faster than $O(h)$, which, however, is just a special behaviour of this particular solution $\mathbf{u}$. On the other hand, the same rate of convergence $O(h)$ for each error should be obtained by using PEERS but, as commented before, employing 50\% more unknowns than our method. Finally, we deduce from the tables that the dominant component of the total error is given by $e(\boldsymbol{\sigma})$, which is actually a quite frequent fact in many mixed finite element schemes. This feature is also evident from the figures, where one sees that the curves corresponding to $e(\boldsymbol{\sigma}, \mathbf{u}, \boldsymbol{\gamma})$ and $e(\boldsymbol{\sigma})$ do not distinguish from each other. 
TABLE 1. Individual and total errors of Example 1 with $\left(\kappa_{1}, \kappa_{2}, \kappa_{3}\right)=\left(\mu, \frac{1}{2 \mu}, \frac{\mu}{2}\right)$ and $\left(\kappa_{1}, \kappa_{2}, \kappa_{3}\right)=\left(\frac{3 \mu}{2}, \frac{1}{4 \mu}, \mu\right)$.

\begin{tabular}{|c|c|c|c|c|c|}
\hline DOF & $h$ & $e(\boldsymbol{\sigma})$ & $e(\mathbf{u})$ & $e(\gamma)$ & $e(\boldsymbol{\sigma}, \mathbf{u}, \boldsymbol{\gamma})$ \\
\hline 643 & 0.12500 & $0.9382 \mathrm{E}-01$ & $5429 \mathrm{E}-02$ & $0.1106 \mathrm{E}-01$ & $0.9463 \mathrm{E}-01$ \\
\hline 1003 & 0.10000 & $0.7576 \mathrm{E}-01$ & $0.3725 \mathrm{E}-02$ & $0.9973 \mathrm{E}-02$ & $0.7650 \mathrm{E}-01$ \\
\hline 1443 & 0.08333 & $0.6345 \mathrm{E}-01$ & $0.2725 \mathrm{E}-02$ & $0.8914 \mathrm{E}-02$ & $0.6413 \mathrm{E}-01$ \\
\hline 1963 & 0.07143 & $0.5455 \mathrm{E}-01$ & $0.2087 \mathrm{E}-02$ & $0.7990 \mathrm{E}-02$ & $0.5517 \mathrm{E}-01$ \\
\hline 2563 & 0.06250 & $0.4782 \mathrm{E}-01$ & $0.1653 \mathrm{E}-02$ & $0.7207 \mathrm{E}-02$ & $0.4 \xi$ \\
\hline 3243 & 0.05556 & $0.4256 \mathrm{E}-01$ & $0.1346 \mathrm{E}-02$ & $0.6545 \mathrm{E}-02$ & $0.4308 \mathrm{E}-01$ \\
\hline 4003 & 0.05000 & $0.3834 \mathrm{E}-01$ & $0.1119 \mathrm{E}-02$ & $0.5984 \mathrm{E}-02$ & 0.38 \\
\hline 4843 & 0.04545 & $0.3488 \mathrm{E}-01$ & $0.9479 \mathrm{E}-03$ & $0.5506 \mathrm{E}-02$ & 0.3 \\
\hline 5763 & 0.04167 & 0.3199E-01 & $0.8151 \mathrm{E}-03$ & $0.5094 \mathrm{E}-02$ & 0.32 \\
\hline 6763 & 0.03846 & $0.2954 \mathrm{E}-01$ & $0.7102 \mathrm{E}-03$ & $0.4736 \mathrm{E}-02$ & 0.2 \\
\hline 7843 & 0.03571 & $0.2744 \mathrm{E}-01$ & $0.6258 \mathrm{E}-03$ & $0.4424 \mathrm{E}-02$ & 0.27 \\
\hline 9003 & 0.03333 & $0.2562 \mathrm{E}-01$ & $0.5569 \mathrm{E}-03$ & 0.4 & 0.2 \\
\hline 10243 & 0.03125 & $0.2402 \mathrm{E}-01$ & $0.5000 \mathrm{E}-03$ & $0.3905 \mathrm{E}-02$ & 0.2 \\
\hline 11563 & 0.02941 & $0.2261 \mathrm{E}-01$ & $0.4523 \mathrm{E}-03$ & $0.3687 \mathrm{E}-02$ & $0.2291 \mathrm{E}-01$ \\
\hline 12963 & 0.02777 & $0.2136 \mathrm{E}-01$ & $0.4120 \mathrm{E}-03$ & $0.3492 \mathrm{E}-02$ & 0.2 \\
\hline 643 & 0.12500 & $0.9377 \mathrm{E}-01$ & $0.2669 \mathrm{E}-02$ & $0.5728 \mathrm{E}-02$ & $\overline{\mathrm{E}-01}$ \\
\hline 1003 & 0.10000 & $0.7572 \mathrm{E}-01$ & $0.1906 \mathrm{E}-02$ & $0.5110 \mathrm{E}-02$ & 0.7 \\
\hline 1443 & 0.08333 & $0.6342 \mathrm{E}-01$ & $0.1441 \mathrm{E}-02$ & $0.4534 \mathrm{E}-02$ & 0.6 \\
\hline 1963 & 0.07143 & $0.5452 \mathrm{E}-01$ & $0.1137 \mathrm{E}-02$ & $0.4043 \mathrm{E}-02$ & 0.54 \\
\hline 2563 & 0.06250 & $0.4780 \mathrm{E}-01$ & $0.9262 \mathrm{E}-03$ & $0.3633 \mathrm{E}-02$ & 0.47 \\
\hline 3243 & 0.05556 & $0.4255 \mathrm{E}-01$ & $0.7744 \mathrm{E}-03$ & $0.3289 \mathrm{E}-02$ & E-01 \\
\hline 4003 & 0.05000 & $0.3833 \mathrm{E}-01$ & $0.6612 \mathrm{E}-03$ & $0.3001 \mathrm{E}-02$ & 0.38 \\
\hline 4843 & 0.04545 & $0.3487 \mathrm{E}-01$ & $0.5745 \mathrm{E}-03$ & $0.2756 \mathrm{E}-02$ & $0.3498 \mathrm{E}-01$ \\
\hline 5763 & 0.04167 & $0.3198 \mathrm{E}-01$ & $0.5065 \mathrm{E}-03$ & $0.2546 \mathrm{E}-02$ & $0.3209 \mathrm{E}-01$ \\
\hline 6763 & 0.03846 & $0.2953 \mathrm{E}-01$ & $0.4520 \mathrm{E}-03$ & $0.2365 \mathrm{E}-02$ & $0.2963 \mathrm{E}-01$ \\
\hline 7843 & 0.03571 & $0.2743 \mathrm{E}-01$ & $0.4076 \mathrm{E}-03$ & $0.2207 \mathrm{E}-02$ & $0.2752 \mathrm{E}-01$ \\
\hline 9003 & 0.03333 & $0.2561 \mathrm{E}-01$ & $0.3708 \mathrm{E}-03$ & $0.2068 \mathrm{E}-02$ & $0.2570 \mathrm{E}-01$ \\
\hline 10243 & 0.03125 & $0.2402 \mathrm{E}-01$ & $0.3400 \mathrm{E}-03$ & $0.1945 \mathrm{E}-02$ & $0.2410 \mathrm{E}-01$ \\
\hline 11563 & 0.02941 & $0.2261 \mathrm{E}-01$ & $0.3137 \mathrm{E}-03$ & $0.1835 \mathrm{E}-02$ & $0.2268 \mathrm{E}-01$ \\
\hline 12963 & 0.02777 & $0.2136 \mathrm{E}-01$ & $0.2913 \mathrm{E}-03$ & $0.1737 \mathrm{E}-02$ & $0.2143 \mathrm{E}-01$ \\
\hline
\end{tabular}

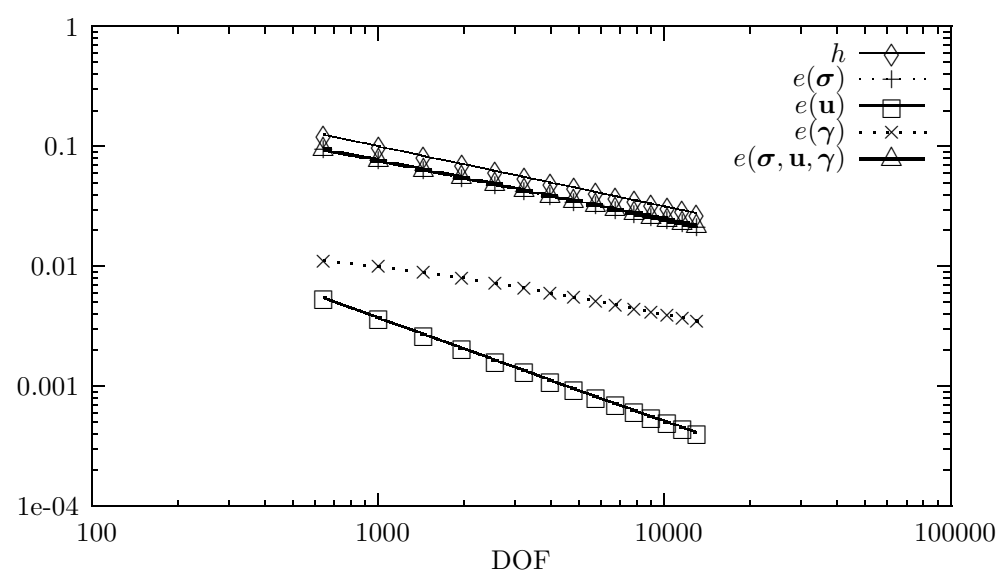

Figure 1. Example 1 with $\left(\kappa_{1}, \kappa_{2}, \kappa_{3}\right)=\left(\mu, \frac{1}{2 \mu}, \frac{\mu}{2}\right)$. 
TABLE 2. Individual and total errors of Example 2 with $\left(\kappa_{1}, \kappa_{2}, \kappa_{3}\right)=\left(\mu, \frac{1}{2 \mu}, \frac{\mu}{2}\right)$ and $\left(\kappa_{1}, \kappa_{2}, \kappa_{3}\right)=\left(\frac{3 \mu}{2}, \frac{1}{4 \mu}, \mu\right)$.

\begin{tabular}{|c|c|c|c|c|c|}
\hline & $h$ & $e(\boldsymbol{\sigma})$ & $e(\mathbf{u})$ & $e(\gamma)$ & $e(\boldsymbol{\sigma}, \mathbf{u}, \boldsymbol{\gamma})$ \\
\hline 643 & 12500 & $134 \mathrm{E}+01$ & $266 \mathrm{E}+00$ & $.1049 \mathrm{E}+01$ & $0.9209 \mathrm{E}+01$ \\
\hline 1003 & 0.10000 & $0.7377 \mathrm{E}+01$ & $0.3602 \mathrm{E}+00$ & $0.9470 \mathrm{E}+00$ & $0.7446 \mathrm{E}+01$ \\
\hline 1443 & 0.08333 & $6179 \mathrm{E}+01$ & $0.2622 \mathrm{E}+00$ & $0.8471 \mathrm{E}+00$ & $0.6242 \mathrm{E}+01$ \\
\hline 1963 & 0.07143 & $0.5313 \mathrm{E}+01$ & $0.1993 \mathrm{E}+00$ & $0.7598 \mathrm{E}+00$ & $0.5370 \mathrm{E}+01$ \\
\hline 2563 & 0.06250 & $0.4658 \mathrm{E}+01$ & $0.1565 \mathrm{E}+00$ & $0.6856 \mathrm{E}+00$ & $0.4710 \mathrm{E}+01$ \\
\hline 3243 & 0.05556 & $0.4146 \mathrm{E}+01$ & $0.1261 \mathrm{E}+00$ & $0.6228 \mathrm{E}+00$ & 0.41 \\
\hline 4003 & 0.05000 & $3735 \mathrm{E}+01$ & $0.1037 \mathrm{E}+00$ & $0.5696 \mathrm{E}+00$ & $0.3779 \mathrm{E}+01$ \\
\hline 4843 & 0.04545 & $0.3397 \mathrm{E}+01$ & $0.8668 \mathrm{E}-01$ & $0.5241 \mathrm{E}+00$ & $0.3438 \mathrm{E}+01$ \\
\hline 5763 & 0.04167 & $0.3116 \mathrm{E}+01$ & $0.7351 \mathrm{E}-01$ & $0.4850 \mathrm{E}+00$ & $0.3154 \mathrm{E}+01$ \\
\hline 6763 & 0.03846 & $0.2877 \mathrm{E}+01$ & 0.6311 & $0.4510 \mathrm{E}+00$ & +01 \\
\hline 7843 & 0.03571 & 0.267 & 0.54 & $\mathrm{E}+00$ & +01 \\
\hline 9003 & 0.03333 & $0.2495 \mathrm{E}+01$ & 0.479 & $0.3951 \mathrm{E}+00$ & 0.25 \\
\hline 10243 & 0.03125 & $0.2340 \mathrm{E}+01$ & 0.4230 & $0.3719 \mathrm{E}+00$ & $0.2369 \mathrm{E}+01$ \\
\hline 11563 & 0.02941 & $0.2202 \mathrm{E}+01$ & 0.3760 & $0.3512 \mathrm{E}+00$ & $0.2230 \mathrm{E}+01$ \\
\hline 12963 & 0.02777 & $0.2080 \mathrm{E}+01$ & $0.3364 \mathrm{E}-01$ & $0.3326 \mathrm{E}+00$ & $0.2107 \mathrm{E}+01$ \\
\hline 643 & 0.12500 & 0.912 & 0.2484 & $0.5489 \mathrm{E}+00$ & $8+01$ \\
\hline 1003 & 0.10000 & $0.7373 \mathrm{E}+01$ & $0.1735 \mathrm{E}+00$ & $0.4903 \mathrm{E}+00$ & $0.7391 \mathrm{E}+01$ \\
\hline 1443 & 0.08333 & $0.6176 \mathrm{E}+01$ & $0.1279 \mathrm{E}+00$ & $0.4354 \mathrm{E}+00$ & $\mathrm{E}+01$ \\
\hline 1963 & 0.07143 & $0.5310 \mathrm{E}+01$ & $0.9789 \mathrm{E}-01$ & $0.3884 \mathrm{E}+00$ & 0.53 \\
\hline 2563 & & & & & +01 \\
\hline 3243 & 0.05556 & $0.4144 \mathrm{E}$ & $0.6233 \mathrm{E}-01$ & $0.3161 \mathrm{E}+00$ & $0.4156 \mathrm{E}+01$ \\
\hline 4003 & 0.05000 & $0.3734 \mathrm{E}+01$ & $0.5131 \mathrm{E}-01$ & $0.2884 \mathrm{E}+00$ & $0.3745 \mathrm{E}+01$ \\
\hline 4843 & 0.04545 & $0.3397 \mathrm{E}+01$ & $0.4294 \mathrm{E}-01$ & $0.2649 \mathrm{E}+00$ & $0.3407 \mathrm{E}+01$ \\
\hline 5763 & 0.04167 & & 0.36431 & $0.2447 \mathrm{E}+00$ & $0.3124 \mathrm{E}+01$ \\
\hline 6763 & 846 & 0.2877 & 0.312 & $0.2273 \mathrm{E}+00$ & $0.2886 \mathrm{E}+01$ \\
\hline 7843 & 0.03571 & $0.2672 \mathrm{E}+01$ & $0.2714 \mathrm{E}-01$ & $0.2121 \mathrm{E}+00$ & $0.2680 \mathrm{E}+01$ \\
\hline 9003 & 0.03333 & $0.2495 \mathrm{E}+01$ & $0.2376 \mathrm{E}-01$ & $0.1988 \mathrm{E}+00$ & $0.2503 \mathrm{E}+01$ \\
\hline 243 & 3125 & & 0.2097 & $0.1870 \mathrm{E}+00$ & \\
\hline 11563 & 0.02941 & $0.2202 \mathrm{E}+01$ & $0.1864 \mathrm{E}-01$ & $0.1764 \mathrm{E}+00$ & $0.2209 \mathrm{E}+01$ \\
\hline 12963 & 0.02777 & $0.2080 \mathrm{E}+01$ & $0.1668 \mathrm{E}-01$ & $0.1670 \mathrm{E}+00$ & $0.2086 \mathrm{E}+01$ \\
\hline
\end{tabular}

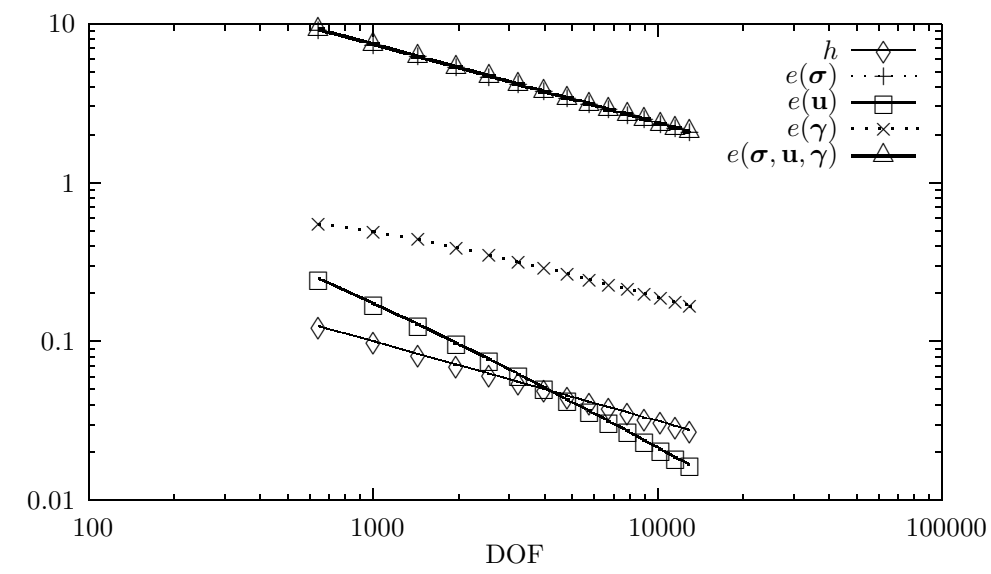

Figure 2. Example 2 with $\left(\kappa_{1}, \kappa_{2}, \kappa_{3}\right)=\left(\frac{3 \mu}{2}, \frac{1}{4 \mu}, \mu\right)$. 
TABLE 3. Individual and total errors of Example 3 with $\left(\kappa_{1}, \kappa_{2}, \kappa_{3}\right)=\left(\mu, \frac{1}{2 \mu}, \frac{\mu}{2}\right)$ and $\left(\kappa_{1}, \kappa_{2}, \kappa_{3}\right)=\left(\frac{3 \mu}{2}, \frac{1}{4 \mu}, \mu\right)$.

\begin{tabular}{|c|c|c|c|c|c|}
\hline $\mathrm{OF}$ & $h$ & $e(\boldsymbol{\sigma})$ & $e(\mathbf{u})$ & $e(\gamma)$ & $e(\boldsymbol{\sigma}, \mathbf{u}, \boldsymbol{\gamma})$ \\
\hline 643 & 22500 & $1671 \mathrm{E}+02$ & $9248 \mathrm{E}+00$ & $0.3978 \mathrm{E}+00$ & $0.1674 \mathrm{E}+02$ \\
\hline 1003 & 0.10000 & $1338 \mathrm{E}+02$ & $0.6676 \mathrm{E}+00$ & $0.2822 \mathrm{E}+00$ & $0.1339 \mathrm{E}+02$ \\
\hline 1443 & 333 & $115 \mathrm{E}+02$ & $5198 \mathrm{E}+00$ & $0.2133 \mathrm{E}+00$ & $0.1116 \mathrm{E}+02$ \\
\hline 1963 & 0.07143 & $0.9563 \mathrm{E}+01$ & $0.4249 \mathrm{E}+00$ & $0.1689 \mathrm{E}+00$ & $0.9573 \mathrm{E}+01$ \\
\hline 2563 & 0.06250 & $0.8369 \mathrm{E}+01$ & $0.3593 \mathrm{E}+00$ & $0.1385 \mathrm{E}+00$ & $0.8377 \mathrm{E}+01$ \\
\hline 3243 & 0.05556 & $0.7440 \mathrm{E}+01$ & $0.3113 \mathrm{E}+00$ & $0.1167 \mathrm{E}+00$ & +01 \\
\hline 4003 & 0.05000 & $697 \mathrm{E}+01$ & $7 \mathrm{E}+00$ & $0.1004 \mathrm{E}+00$ & $0.6703 \mathrm{E}+01$ \\
\hline 4843 & 0.04545 & $0.6088 \mathrm{E}+01$ & $0.2459 \mathrm{E}+00$ & $0.8795 \mathrm{E}-01$ & $0.6093 \mathrm{E}+01$ \\
\hline 5763 & 0.04167 & $0.5581 \mathrm{E}+01$ & $0.2227 \mathrm{E}+00$ & $0.7810 \mathrm{E}-01$ & $0.5585 \mathrm{E}+01$ \\
\hline 6763 & 0.03846 & $5152 \mathrm{E}+01$ & $0.2035 \mathrm{E}+00$ & $0.7016 \mathrm{E}-01$ & $0.5156 \mathrm{E}+01$ \\
\hline 7843 & 571 & $784 \mathrm{E}+01$ & 0.1 & 0.63 & +01 \\
\hline 9003 & 0.03333 & 0.446 & & -01 & +01 \\
\hline 10243 & 0.03125 & -01 & +00 & $0.5362 \mathrm{E}-01$ & +01 \\
\hline 11563 & 0.02941 & $0.3940 \mathrm{E}+01$ & $0.1517 \mathrm{E}+00$ & 0.4970 & +01 \\
\hline 12963 & 0.02777 & $0.3721 \mathrm{E}+01$ & $0.1427 \mathrm{E}+00$ & $0.4630 \mathrm{E}-01$ & $0.3724 \mathrm{E}+01$ \\
\hline 643 & & & & $0.2323 \mathrm{E}+00$ & $0.1672 \mathrm{E}+02$ \\
\hline 1003 & 0.10000 & -02 & $\mathrm{E}+00$ & $0.1745 \mathrm{E}+00$ & $\mathrm{E}+02$ \\
\hline 1443 & 0.08333 & $0.1115 \mathrm{E}+02$ & $0.4324 \mathrm{E}+00$ & $0.1392 \mathrm{E}+00$ & $0.1115 \mathrm{E}+02$ \\
\hline 1963 & 0.07143 & $0.9562 \mathrm{E}+01$ & $0.3664 \mathrm{E}+00$ & $0.1157 \mathrm{E}+00$ & $0.9569 \mathrm{E}+01$ \\
\hline 2563 & & & & & \\
\hline 3243 & 0.05556 & $0.7439 \mathrm{E}+01$ & $0.2812 \mathrm{E}+00$ & $0.8652 \mathrm{E}-01$ & +01 \\
\hline 4003 & 0.05000 & $0.6696 \mathrm{E}+01$ & $0.2520 \mathrm{E}+00$ & $0.7685 \mathrm{E}-01$ & $0.6701 \mathrm{E}+01$ \\
\hline 4843 & 0.04545 & $0.6088 \mathrm{E}+01$ & $0.2284 \mathrm{E}+00$ & $0.6914 \mathrm{E}-01$ & $0.6092 \mathrm{E}+01$ \\
\hline 5763 & & & & $0.6285 \mathrm{E}-01$ & \\
\hline 6763 & 0.03846 & 0.515 & 0.19 & $0.5762 \mathrm{E}-01$ & $\mathrm{E}+01$ \\
\hline 7843 & 0.03571 & $0.4784 \mathrm{E}+01$ & $0.1783 \mathrm{E}+00$ & $0.5320 \mathrm{E}-01$ & $0.4787 \mathrm{E}+01$ \\
\hline 9003 & 0.03333 & 0.4465 & $0.1662 \mathrm{E}+00$ & $0.4942 \mathrm{E}-01$ & $\mathrm{E}+01$ \\
\hline 0243 & & & & & \\
\hline 11563 & 0.02941 & $0.3940 \mathrm{E}+01$ & $0.1464 \mathrm{E}+00$ & $0.4329 \mathrm{E}-01$ & $0.3941 \mathrm{E}+01$ \\
\hline 12963 & 0.02777 & $0.3721 \mathrm{E}+01$ & $0.1381 \mathrm{E}+00$ & $0.4076 \mathrm{E}-01$ & $0.3723 \mathrm{E}+01$ \\
\hline
\end{tabular}

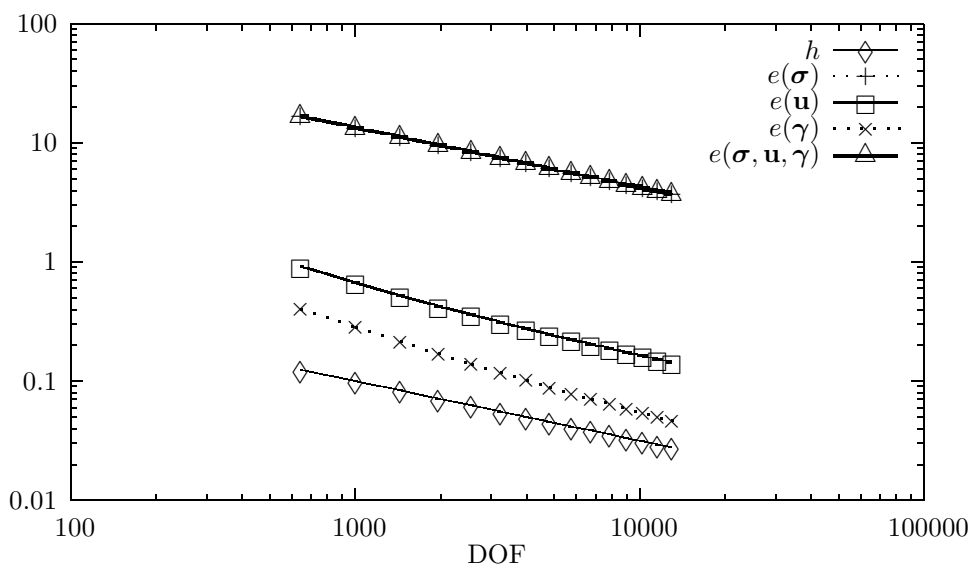

Figure 3. Example 3 with $\left(\kappa_{1}, \kappa_{2}, \kappa_{3}\right)=\left(\mu, \frac{1}{2 \mu}, \frac{\mu}{2}\right)$. 
TABLE 4. Individual and total errors of Example 4 with $\left(\kappa_{1}, \kappa_{2}, \kappa_{3}\right)=\left(\mu, \frac{1}{2 \mu}, \frac{\mu}{2}\right)$ and $\left(\kappa_{1}, \kappa_{2}, \kappa_{3}\right)=\left(\frac{3 \mu}{2}, \frac{1}{4 \mu}, \mu\right)$.

\begin{tabular}{|c|c|c|c|c|c|}
\hline $\mathrm{OF}$ & $h$ & $e(\boldsymbol{\sigma})$ & $e(\mathbf{u})$ & $e(\gamma)$ & $e(\boldsymbol{\sigma}, \mathbf{u}, \boldsymbol{\gamma})$ \\
\hline 643 & 22500 & $631 \mathrm{E}+04$ & $6712 \mathrm{E}+02$ & $.4018 \mathrm{E}+02$ & $0.1633 \mathrm{E}+04$ \\
\hline 1003 & 10000 & $1306 \mathrm{E}+04$ & $0.4427 \mathrm{E}+02$ & $0.2774 \mathrm{E}+02$ & $0.1307 \mathrm{E}+04$ \\
\hline 1443 & 333 & $9 \mathrm{E}+04$ & $0 \Gamma$ & $.2028 \mathrm{E}+02$ & $0.1090 \mathrm{E}+04$ \\
\hline 1963 & 0.07143 & $9334 \mathrm{E}+03$ & $0.2378 \mathrm{E}+02$ & $0.1548 \mathrm{E}+02$ & $0.9338 \mathrm{E}+03$ \\
\hline 2563 & 0.06250 & $0.8169 \mathrm{E}+03$ & $0.1860 \mathrm{E}+02$ & $0.1221 \mathrm{E}+02$ & $0.8172 \mathrm{E}+03$ \\
\hline 3243 & 0.05556 & $262 \mathrm{E}+03$ & $0.1498 \mathrm{E}+02$ & $0.9892 \mathrm{E}+01$ & 0.726 \\
\hline 4003 & 5000 & $36 \mathrm{~F}+03$ & $34 \mathrm{E}+02$ & & 0.65 \\
\hline 4843 & 0.04545 & $5942 \mathrm{E}+03$ & $0.1036 \mathrm{E}+02$ & $0.6886 \mathrm{E}+01$ & $0.5943 \mathrm{E}+03$ \\
\hline 5763 & 0.04167 & $0.5447 \mathrm{E}+03$ & $0.8824 \mathrm{E}+01$ & $0.5879 \mathrm{E}+01$ & $0.5448 \mathrm{E}+03$ \\
\hline 6763 & 0.03846 & $5028 \mathrm{E}+03$ & $0.7613 \mathrm{E}+01$ & $0.5081 \mathrm{E}+01$ & 0.50 \\
\hline 7843 & 0. & +03 & 0.6 & & \\
\hline 9003 & 0.03 & & 0.58 & & \\
\hline 10243 & 0.03125 & +03 & +01 & -01 & 0.4 \\
\hline 11563 & 0.02941 & $0.3846 \mathrm{E}+03$ & $0.4637 \mathrm{E}+01$ & $0.3108 \mathrm{E}+01$ & +03 \\
\hline 12963 & 0.02777 & $0.3632 \mathrm{E}+03$ & $0.4171 \mathrm{E}+01$ & $0.2797 \mathrm{E}+01$ & 0.363 \\
\hline 643 & & & & $0.1994 \mathrm{E}+02$ & 0.16 \\
\hline 1003 & 0.10000 & +04 & 0.20 & $0.1367 \mathrm{E}+02$ & 0.13 \\
\hline 1443 & 0.08333 & $E+04$ & $\mathrm{E}+02$ & $0.9966 \mathrm{E}+01$ & $\mathrm{E}+04$ \\
\hline 1963 & 0.07143 & $0.9333 \mathrm{E}+03$ & $\mathrm{E}+02$ & $0.7597 \mathrm{E}+01$ & $4 \mathrm{E}+03$ \\
\hline 2563 & & & & & \\
\hline 3243 & 0.05556 & -03 & $0.7186 \mathrm{E}+01$ & $0.4852 \mathrm{E}+01$ & 0.726 \\
\hline 4003 & 0.05000 & $0.6536 \mathrm{E}+03$ & $0.5958 \mathrm{E}+01$ & $0.4015 \mathrm{E}+01$ & $0.6536 \mathrm{E}+03$ \\
\hline 4843 & 0.04545 & $0.5942 \mathrm{E}+03$ & $0.5026 \mathrm{E}+01$ & $0.3380 \mathrm{E}+01$ & $0.5942 \mathrm{E}+03$ \\
\hline 5763 & & & & & \\
\hline 6763 & 0.0 & 03 & 0.37 & 0.2496 & 0.50 \\
\hline 7843 & 0.03571 & $0.4669 \mathrm{E}+03$ & $0.3260 \mathrm{E}+01$ & $0.2180 \mathrm{E}+01$ & $0.4669 \mathrm{E}+03$ \\
\hline 9003 & 0.03333 & 0.4358 & $0.2878 \mathrm{E}+01$ & 0.1922 & 0.43 \\
\hline 0243 & & & & & \\
\hline 11563 & 0.02941 & $0.3845 \mathrm{E}+03$ & $0.2294 \mathrm{E}+01$ & $0.1529 \mathrm{E}+01$ & $0.3845 \mathrm{E}+03$ \\
\hline 12963 & 0.02777 & $0.3632 \mathrm{E}+03$ & $0.2068 \mathrm{E}+01$ & $0.1376 \mathrm{E}+01$ & $0.3632 \mathrm{E}+03$ \\
\hline
\end{tabular}

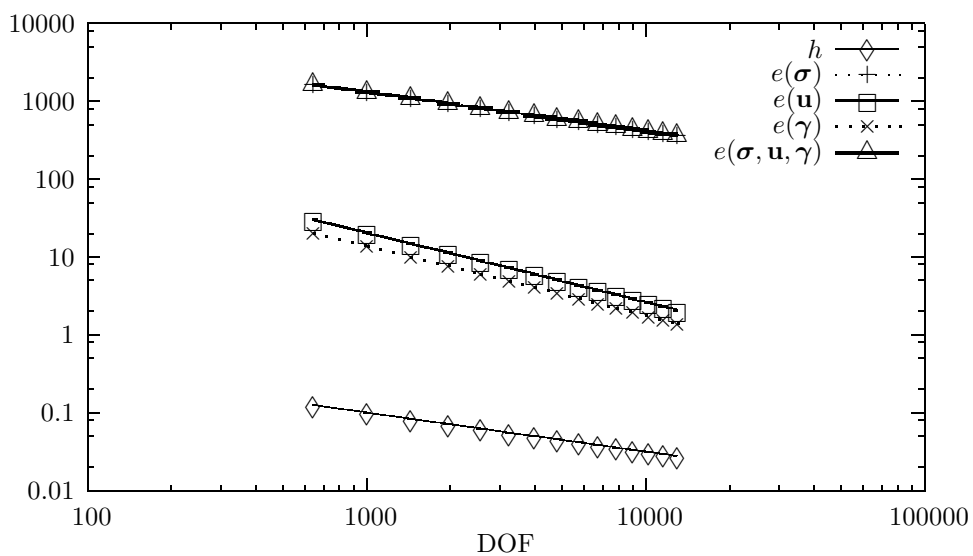

Figure 4. Example 4 with $\left(\kappa_{1}, \kappa_{2}, \kappa_{3}\right)=\left(\frac{3 \mu}{2}, \frac{1}{4 \mu}, \mu\right)$. 
Summarizing, the numerical results presented here constitute enough support for our augmented mixed finite element scheme to be considered as a valid and competive alternative when solving the linear elasticity problem. We end this section by mentioning that further developments on this approach, including other computational aspects, preconditioning of (4.42), a posteriori error analysis, adaptivity, and corresponding numerical experiments with larger numbers of degrees of freedom, will be reported in separate works.

Acknowledgements. The author is thankful to the editor and the referees for valuable remarks and suggestions that improved the paper. Also, he expresses his gratitude to Norbert Heuer and Tomás P. Barrios for performing the computational code and running the numerical examples, respectively.

\section{REFERENCES}

[1] D.N. Arnold, F. Brezzi and J. Douglas, PEERS: A new mixed finite element method for plane elasticity. Japan J. Appl. Math. 1 (1984) 347-367.

[2] D.N. Arnold, F. Brezzi and M. Fortin, A stable finite element method for the Stokes equations. Calcolo 21 (1984) $337-344$.

[3] D.N. Arnold, J. Douglas and Ch.P. Gupta, A family of higher order mixed finite element methods for plane elasticity. Numer. Math. 45 (1984) 1-22.

[4] D. Arnold and R. Falk, Well-posedness of the fundamental boundary value problems for constrained anisotropic elastic materials. Arch. Rational Mech. Analysis 98 (1987) 143-190.

[5] I. Babuška and G.N. Gatica, On the mixed finite element method with Lagrange multipliers. Numer. Methods Partial Differential Equations 19 (2003) 192-210.

[6] M. Barrientos, G.N. Gatica and E.P. Stephan, A mixed finite element method for nonlinear elasticity: two-fold saddle point approach and a posteriori error estimate. Numer. Math. 91 (2002) 197-222.

[7] T.P. Barrios, G.N. Gatica and F. Paiva, A wavelet-based stabilization of the mixed finite element method with Lagrange multipliers. Appl. Math. Lett. (in press).

[8] D. Braess, Finite Elements. Theory, Fast Solvers, and Applications in Solid Mechanics. Cambridge University Press (1997).

[9] F. Brezzi and J. Douglas, Stabilized mixed methods for the Stokes problem. Numer. Math. 53 (1988) 225-235.

[10] F. Brezzi and M. Fortin, Mixed and Hybrid Finite Element Methods. Springer-Verlag (1991).

[11] F. Brezzi and M. Fortin, A minimal stabilisation procedure for mixed finite element methods. Numer. Math. 89 (2001) $457-491$.

[12] F. Brezzi, J. Douglas and L.D. Marini, Variable degree mixed methods for second order elliptic problems. Mat. Apl. Comput. 4 (1985) 19-34.

[13] F. Brezzi, J. Douglas and L.D. Marini, Two families of mixed finite elements for second order elliptic problems. Numer. Math. 47 (1985) 217-235.

[14] F. Brezzi, M. Fortin and L.D. Marini, Mixed finite element methods with continuous stresses. Math. Models Methods Appl. Sci. 3 (1993) 275-287.

[15] D. Chapelle and R. Stenberg, Locking-free mixed stabilized finite element methods for bending-dominated shells, in Plates and shells (Quebec, QC, 1996), American Mathematical Society, Providence, RI, CRM Proceedings Lecture Notes 21 (1999) 81-94.

[16] P.G. Ciarlet, The Finite Element Method for Elliptic Problems. North-Holland (1978).

[17] J. Douglas and J. Wan, An absolutely stabilized finite element method for the Stokes problem. Math. Comput. 52 (1989) 495-508.

[18] H.-Y. Duan and G.-P. Liang, Analysis of some stabilized low-order mixed finite element methods for Reissner-Mindlin plates. Comput. Methods Appl. Mech. Engrg. 191 (2001) 157-179.

[19] L.P. Franca, New Mixed Finite Element Methods. Ph.D. Thesis, Stanford University (1987).

[20] L.P. Franca and T.J.R. Hughes, Two classes of finite element methods. Comput. Methods Appl. Mech. Engrg. 69 (1988) 89-129.

[21] L.P. Franca and A. Russo, Unlocking with residual-free bubbles. Comput. Methods Appl. Mech. Engrg. 142 (1997) 361-364.

[22] L.P. Franca and R. Stenberg, Error analysis of Galerkin least squares methods for the elasticity equations. SIAM J. Numer. Anal. 28 (1991) 1680-1697.

[23] C.O. Horgan, Korn's inequalities and their applications in continuum mechanics. SIAM Rev. 37 (1995) 491-511.

[24] C.O. Horgan and J.K. Knowles, Eigenvalue problems associated with Korn's inequalities. Arch. Rational Mech. Anal. 40 (1971) 384-402.

[25] C.O. Horgan and L.E. Payne, On inequalities of Korn, Friedrichs and Babuška-Aziz. Arch. Rational Mech. Analysis 82 (1983) $165-179$.

[26] N. Kechkar and D. Silvester, Analysis of locally stabilized mixed finite element methods for the Stokes problem. Math. Comput. $58(1992) 1-10$. 
[27] G. Lube and A. Auge, Stabilized mixed finite element approximations of incompressible flow problems. Zeitschrift für Angewandte Mathematik und Mechanik 72 (1992) T483-T486.

[28] W. McLean, Strongly Elliptic Systems and Boundary Integral Equations. Cambridge University Press (2000).

[29] A. Masud and T.J.R. Hughes, A stabilized mixed finite element method for Darcy flow. Comput. Methods Appl. Mech. Engrg. 191 (2002) 4341-4370.

[30] R. Nascimbene and P. Venini, A new locking-free equilibrium mixed element for plane elasticity with continuous displacement interpolation. Comput. Methods Appl. Mech. Engrg 191 (2002) 1843-1860.

[31] S. Norburn and D. Silvester, Fourier analysis of stabilized $Q_{1}-Q_{1}$ mixed finite element approximation. SIAM J. Numer. Anal. 39 (2001) 817-833.

[32] R. Stenberg, A family of mixed finite elements for the elasticity problem. Numer. Math. 53 (1988) 513-538.

[33] T. Zhou, Stabilized hybrid finite element methods based on the combination of saddle point principles of elasticity problems. Math. Comput. 72 (2003) 1655-1673.

[34] T. Zhou and L. Zhou, Analysis of locally stabilized mixed finite element methods for the linear elasticity problem. Chinese J. Engrg Math. 12 (1995) 1-6. 